\title{
CROSSED PRODUCTS AND RAMIFICATION
}

\author{
SUSAN WILLIAMSON
}

Introduction. Let $S$ be the integral closure of a complete discrete rank one valuation ring $R$ in a finite Galois extension of the quotient field of $R$, and let $G$ denote the Galois group of the quotient field extension. Auslander and Rim have shown in [3] that the trivial crossed product $\Delta(1, S, G)$ is an hereditary order if and only if $S$ is a tamely ramified extension of $R$. And the author has proved in [7] that if the extension $S$ of $R$ is tamely ramified then the crossed product $\Delta(f, S, G)$ is a $\Pi$-principal hereditary order for each 2cocycle $f$ in $Z^{2}(G, U(S))$. (See Section 1 for the definition of $\Pi$-principal hereditary order.) However, the author has exhibited in [8] an example of a crossed product $\Delta(f, S, G)$ which is a $\Pi$-principal hereditary order in the case when $S$ is a wildly ramified extension of $R$. The purpose of this paper is to present necessary and sufficient conditions for a crossed product $\Delta(f, S, G)$ to be a $\Pi$-principal hereditary order when the extension $S$ of $R$ has a separable residue class field extension.

Let $S$ be an extension of $R$ (with separable residue class field extension) and let $C$ denote the center of the first ramification group. In Section 1 we define for each element $[f]$ of $H^{2}(G, U(S))$ a subgroup $R_{f}$ of $C$ called the radical group of $[f]$. The main theorem of the paper states that the crossed product $\Delta(f, S, G)$ is a $\Pi$-principal hereditary order if and only if the radical group of $[f]$ is trivial. As a corollary we obtain the result of Harada (see [10]) that if $R$ has perfect residue class field, then $\Delta(f, S, G)$ is a $\Pi$-principal hereditary order if and only if $S$ is a tamely ramified extension of $R$. In an appendix we present some facts concerning the cohomology of groups which shall be referred to in the paper.

The following notation shall be employed throughout the entire paper. If $R$ is a ring then its multiplicative group of units shall be denoted by $U(R)$, and its radical by $\operatorname{rad} R$. If $R$ is a local ring, then $\bar{R}$ shall denote its residue 
class field. Unless otherwise stated, $S$ shall always denote the integral closure of a complete discrete rank one valuation ring $R$ in a finite Galois extension of the quotient field of $R$, and $G$ the Galois group of the quotient field extension. Since $R$ is complete, $S$ is also a complete discrete rank one valuation ring, and $I I$ shall denote a prime element of $S$. The $i^{\text {th }}$ ramification group of the extension $S$ of $R$ shall be denoted by $G_{i}$. That it to say, $G_{i}$ is the set of all elements $\sigma$ of $G$ such that $\sigma(s) \equiv s \bmod \Pi^{i+1}$ for all $s$ in $S$. Each group $G_{i}$ is a normal subgroup of $G$, and the inertia group $G_{0}$ acts trivially on $\bar{S}$.

More generally, if $G$ is a finite group and $A$ is a $G$-ring over a unitary commutative ring $R$, then $[f]$ shall denote the cohomology class in $H^{2}(G$, $U(A)$ ) of the 2-cocycle $f$ of $Z^{2}(G, U(A))$. Furthermore, if $A$ is a local ring, then $\vec{f}$ shall denote the image of $f$ under the natural map $Z^{2}(G, U(A)) \rightarrow Z^{2}(G$, $U(\bar{A}))$. For the definitions of crossed product and hereditary order we refer the reader to [7]. The definitions of tame ramification and wild ramification are given on pp. 88-89 of [6].

For the convenience of the reader we summarize some important facts about ramification groups which may be found in Chapter IV of [5]. Let $S$ denote the integral closure of a complete discrete rank one valuation ring $R$ in a finite Galois extension of the quotient field of $R$ such that the residue class field extension is separable. If $\bar{R}$ has characteristic zero then the first ramification group vanishes. If the characteristic $p$ of $\bar{R}$ is non-zero then $G_{1}$ is a $p$-group. The factor group $G_{0} / G_{1}$ is a cyclic group whose order is relatively prime to $p$. For $i \geq 1$, each factor group $G_{i} / G_{i+1}$ is an Abelian $p$-group of type $(p, p, \ldots, p)$.

1. The radical group. Let $S$ denote the integral closure of a complete discrete rank one valuation ring $R$ in a finite Galois extension $K$ of the quotient field $k$ of $R$, and let $G$ denote the Galois group of $K$ over $k$. If $[f]$ is an element of $H^{2}(G, U(S))$ then the crossed product $\Delta(f, S, G)$ is an $R$-order in the central simple $k$-algebra $\Delta(f, K, G)$. If $I$ denotes a prime element of $S$ it is easy to verify from the definition of crossed product that the left ideal $\Delta(f, S, G) \Pi$ of $\Delta(f, S, G)$ is in fact a two-sided ideal. Therefore $\Pi$ is always contained in the radical of $\Delta(f, S, G)$ according to Lemma 1.4. In the case when $\Delta(f, S, G) \Pi$ is precisely the radical of $\Delta(f, S, G)$ we may conclude that the crossed product $\Delta(f, S, G)$ is an hereditary order by the Corollary to 
Theorem 2.2 of [2], since $\Delta(f, S, G) \Pi$ is a free left $\Delta(f, S, G)$-module. This leads us to make the following definition.

Definition. A crossed product $\Delta(f, S, G)$ is called a II-principal hereditary order if its radical is generated by the prime element $I I$ of $S$.

We have already established the existence of a large class of $\Pi$-principal hereditary orders, namely each crossed product $\Delta(f, S, G)$ in the case when $S$ is a tamely ramified extension of $R$. The purpose of this paper is to study $\Pi$-principal hereditary orders in the more general case when the residue class field extension is separable. Observe that the crossed product $\Delta(f, S, G)$ is a $\Pi$-principal hereditary order if and only if the crossed product $\Delta(\bar{f}, \bar{S}, G)$ is a semi-simple ring.

Let $S$ be an extension of $R$ with $\bar{S}$ separable over $\bar{R}$, and let $C$ denote the center of the first ramification group. In Sections 2 and 3 the question of the semi-simplicity of $\Delta(\bar{f}, \bar{S}, G)$ shall be reduced to the question of the semisimplicity of $\Delta(\bar{f}, \bar{S}, C)$.

Therefore our object of study in Section 1 is the crossed product $\Delta(f, F, C)$ where $C$ is an Abelian $p$-group which acts trivially on a field $F$ of characteristic p. We shall define for each element $[f]$ of $H^{2}(C, U(F))$ a subgroup $R_{f}$ of $C$ and prove that $\Delta(f, F, C)$ is semi-simple if and only if $R_{f}$ is trivial. The following remark establishes notation which shall be in constant use throughout this section.

Remark 1.1. Let $C=E_{1} \times \cdots \times E_{t}$ be a decomposition of an Abelian $p$-group $C$ into a direct product of cyclic $p$-groups. It is well known that such a decomposition of $C$ is unique up to isomorphism except for the order of the cyclic components, (see Theorem 3.3.2 of [4]). Let $F$ be a field of characteristic $p$ such that $C$ acts trivially on $F$. If $[f]$ is an element of $H^{2}(C, U(F))$ we may assume according to Cor. A. 3 that $f$ has been normalized in the sense of Abelian $p$-groups, so that $f=f_{1} \cdots f_{t}$ where each element $f_{i}$ of $Z^{2}\left(E_{i}, U(F)\right)$ is normalized in the sense of cyclic groups. The symbol $h_{i}(X)$ for $1 \leq i \leq t$ shall denote the polynomial $h_{i}(X)=X^{e_{i}}-a_{i}$ in $F[X]$ where $e_{i}$ is the order of $E_{i}$, and $a_{i}$ is an element of $U(F)$ such that $\left[f_{i}\right]$ corresponds to $a_{i} \bmod [U(F)]^{e_{i}}$ under the canonical identification $H^{2}\left(E_{i}, U(F)\right)=U(F) /[U(F)]^{e_{i}}$.

We next observe that the crossed product $\Delta(f, F, C)$ is isomorphic to a 
tensor product over $F$ of factor rings of the polynomial ring $F[X]$.

Proposition 1.2. The crossed product $\Delta(f, F, C)$ is F-algebra isomorphic to the tensor product $A_{1} \otimes A_{2} \otimes \cdots \otimes A_{t}$ over $F$ where $A_{i}=F[X] /\left(h_{i}(X)\right)$ for $1 \leq i \leq t$.

Proof. The proof is by induction on the number $t$ of cyclic components of the Abelian $p$-group $C$. If $t=1$, then $C$ is cyclic. Since $f$ is normalized in the sense of cyclic groups, the map $\psi: \Delta(f, F, C) \rightarrow F[X] /\left(h_{1}(X)\right)$ induced by defining $\phi\left(u_{s}\right)=X$ is an $F$-algebra isomorphism where $\sigma$ denotes a generator of $C$.

For the inductive step we now suppose that $C$ has $n$ cyclic components, say $C=E_{1} \times \cdots \times E_{n-1} \times E_{n}$, and consider the subgroup $C_{n-1}=E_{1} \times \cdots \times E_{n-1}$. Then $\Delta(f, F, C)=\Delta\left(g f_{n}, F, C_{n-1} \times E_{n}\right)$ where $g=f_{1} \cdots f_{n-1}$. Using the fact that $f$ is normalized in the sense of Abelian p-groups one can easily verify that the natural map.

$$
\psi: \Delta\left(g f_{n}, F, C_{n-1} \times E_{n}\right) \rightarrow \Delta\left(g, F, C_{n-1}\right) \otimes_{F} \Delta\left(f_{n}, F, E_{\eta}\right)
$$

is an $F$-algebra isomorphism. The induction hypothesis states that the assertion of the proposition is true for Abelian $p$-groups with $n-1$ cyclic components. Therefore the crossed product $\Delta\left(g, F, C_{n-1}\right)$ is $F$-algebra isomorphic to $A_{1} \otimes \cdots \otimes A_{n-1}$. And since $E_{n}$ is cyclic, it follows from the first part of the proof that $\Delta\left(f_{n}, F, E_{n}\right)$ is $F$-algebra isomorphic to $A_{n}$. Combining these results we conclude that $\Delta(f, F, C)$ is $F$-algebra isomorphic to $A_{1} \otimes \cdots \otimes A_{n}$.

The next object is to establish a criterion for the semi-simplicity of $\Delta(f, F, C)$ in terms of the irreducibility of the polynomials $h_{i}(X)$ and thus establish a connection between the semi-simplicity of $\Delta(f, F, C)$ and cohomology. In order to do this we first prove two lemmas.

Lemma 1.3. Let $F$ be a field, and let $H(X)$ be a non-constant polynomial in $F[X]$. Denote the factor ring $F[X] /(H(X))$ by $L_{2}$. If $L_{1}$ is a field containing $F$, then the tensor product $L_{1} \otimes_{F} L_{2}$ is $L_{1}$-algebra isomorphic to $L_{1}[Y] /(H(Y))$.

Proof. Define the map $\varphi: L_{1} \otimes L_{2} \rightarrow L_{1}[Y] /(H(Y))$ by $\varphi\left(\sum a_{i} \otimes f_{i}(X) / H(X)\right)$ $=\sum a_{i} f_{i}(Y) /(H(Y))$ where the $a_{i}$ are in $L_{1}$ and the $f_{i}$ are in $F[X]$. If is easy to verify that $\varphi$ is a well-defined $L_{1}$-algebra epimorphism.

In order to prove that $\varphi$ is a monomorphism we first observe that $\left(1, X, \ldots, X^{n-1}\right)$ is a generating set for $L_{2}$ over $F$ where $n$ is the degree of 
$H(X)$. Therefore any element of $L_{1} \otimes L_{2}$ may be written in the form $\sum_{i=0}^{n-1} a_{i} \otimes X^{i} /(H(X))$ where the $a_{i}$ are in $L_{1}$. Suppose now that $\sum a_{i} \otimes X^{i} /(H(X))$ is in the kernel of $\varphi$. Then the equalities $\varphi\left(\sum a_{i} \otimes X^{i} /(H(X))\right)=\sum a_{i} Y^{i} /(H(Y))=0$ imply that the polynomial $\sum_{i=0}^{n-1} a_{i} Y^{i}$ of $L_{1}[Y]$ is in the principal ideal generated by $H(Y)$, so that $\sum a_{i} Y^{i}=g(Y) H(Y)$ for some element $g(Y)$ of $L_{1}[Y]$. Now the degree of $\sum_{i=0}^{n-1} a_{i} Y^{i}$ is less than or equal to $n-1$. However, the degree of $g(Y) H(Y)$ is less than $n$ if and only if. $g(Y)$ is the zero polynomial since $H(Y)$ has degree $n$ and $L_{1}$ is a field. Therefore $g(Y)$ is the zero polynomial, and so the equality $\sum a_{i} Y^{i}=g(Y) H(Y)$ implies that $a_{i}=0$ for $0 \leq i \leq n-1$. Therefore $\operatorname{ker} \varphi=(0)$ and so $\varphi$ is a monomorphism.

LEMmA 1.4. Let the extension $S$ of $R$ be a ring extension. If $S$ is a finitely generated left $R$-module and $(\mathrm{rad} R) S=S(\mathrm{rad} R)$ then $\mathrm{rad} R$ is contained in rad S.

Proof. To show that $\operatorname{rad} R$ is contained in $\operatorname{rad} S$ it suffices to show that if $M$ is a finitely generated left $S$-module and $S(\operatorname{rad} R) M=M$, then $M=0$. The fact that $S(\operatorname{rad} R)=(\operatorname{rad} R) S$ implies that $S(\operatorname{rad} R) M=(\operatorname{rad} R) S M=$ $(\operatorname{rad} R) M$. Since $M$ is a finitely generated left $S$-module and $S$ is a finitely generated left $R$-module it follows that $M$ is a finitely generated left $R$-module. Therefore the equality $(\operatorname{rad} R) M=M$ implies that $M=(0)$. Hence $S(\operatorname{rad} R)$ is contained in $\mathrm{rad} S$.

Proposition 1.5. Let $F$ be a field of characteristic $p \neq 0$, and let $H_{i}(X)$ for $1 \leq i \leq t$ be elements of $F[X]$ of the form $H_{i}(X)=X^{e_{i}}-a_{i}$ where each $e_{i}$ is a $p^{t h}$ power. Let $A$ denote the tensor product $L_{1} \otimes \cdots \otimes L_{t}$ over $F$ where $L_{i}=F[X] /\left(H_{i}(X)\right)$. Then the following statements are equivalent

1) $A$ is semi-simple

2) $A$ is a field

3) each polynomial $H_{i}(X)$ is irreducible in a splitting field for $\prod_{j<i} H_{j}(X)$ over F.

Proof. The proof is by induction on the number $t$ of polynomials $H_{i}(X)$. We first prove that the statements are equivalent when $t=1$. In this case $A$ is of the form $A=F[X] /(H(X))$ where $H(X)=X^{e}-a$ and $e$ is a $p^{t h}$ power. Since $F$ has characteristic $p$, a factorization of $H(X)$ into a product of irreducible 
polynomials of $F[X]$ is of the form $H(X)=\left(X^{m}-b\right)^{e / m}$ where $b^{e / m}=a$ and $m$ is a divisor of $e$. The radical of the commutative Artin ring $F[X] /\left(X^{e}-a\right)$ is generated by the residue class of the polynomial $X^{m}-b$. Therefore $A$ is semi-simple if and only if $m=e$, that is if and only if $H(X)$ is irreducible in $F[X]$. Therefore 1$)$ is equivalent to 3$)$. However, the polynomial $H(X)$ is irreducible in $F[X]$ if and only if $F[X] /(H(X))$ is a field. Therefore 3$)$ is equivalent to 2) and this completes the proof in the case when $t=1$.

For the inductive step we assume the equivalence of the statements for $t<n$, and prove their equivalence for $t=n$. Throughout the rest of the proof it shall be convenient to use the notation $A_{i}=L_{1} \otimes \cdots \otimes L_{i}$ for $1 \leq i \leq n$, and $A_{0}=F$.

We show first that 1) implies 2). So suppose that $A=A_{n}$ is semi-simple. Using the induction hypothesis we shall prove that each $A_{i}$ for $1 \leq i \leq n-1$ must be a field. Observe that the natural map $A_{i} \rightarrow A$ is an injection because each $A_{i}$ is a free $F$-module. Since $A$ is a finitely generated commutative $A_{i}$ algebra, the radical of $A_{i}$ is contained in the radical of $A$ according to Lemma 1.4. From the semi-simplicity of $A$ we conclude that $A_{i}$ has zero radical. Hence $A_{i}$ is semi-simple because it is an Artin ring. The induction hypothesis implies therefore that $A_{i}$ is a field for $1 \leq i \leq n-1$.

Now we may prove that 1) implies 2). For since $A_{n-1}$ is a field, and $A=A_{n-1} \otimes L_{n}$, we know that $A$ is $A_{n-1}$-algebra isomorphic to $A_{n-1}[Y] /\left(Y^{e_{n}}-a_{n}\right)$ by Lemma 1.3. Thus we have reduced the problem to the case $t=1$, and so the assumption that $A$ is semi-simple implies that $A$ is a field.

In order to prove that 1) implies 3 ) we first observe that if $A$ is semisimple, then each $A_{i}$ for $1 \leq i \leq n$ is a splitting field over $F$ for the polynomial $\sum_{j \leq i} H_{j}(X)$. For by the above, the semi-simplicity of $A$ implies that each $A_{i}$ for $1 \leq i \leq n$ is a field and is therefore $A_{i-1}$-algebra isomorphic to $A_{i-1}[Y] /\left(H_{i}(Y)\right)$ according to Lemma 1.3. It now follows easily by induction that $A_{i}$ is a splitting field for $\prod_{j \leq \imath} H_{j}(X)$ over $F$. Now we may prove that 1) implies 3). For the fact that $A_{i}$ is $A_{i-1}$-algebra isomorphic to $A_{i-1}[Y] /\left(Y^{e_{i}}-a_{i}\right)$ together with the fact that $A_{i}$ is a field implies that $H_{i}(Y)$ is irreducible over $A_{i-1}$ which is a splitting field for $\prod_{j<i} H_{j}(X)$.

We prove next that 3 ) implies 2). Consider the $n$ polynomials $H_{i}(X)$ and assume that each polynomial $H_{i}(X)$ is irreducible in a splitting field for $\prod_{j<i} H_{j}(X)$. 
Then certainly each $H_{i}(X)$ for $i<n$ is irreducible in a splitting field for $\prod_{j<i} H_{j}(X)$. By the induction hypothesis we may conclude therefore that $A_{n-1}$ is a field. We have already shown that if $A_{n-1}$ is a field it is necessarily a splitting field for $\prod_{\jmath<n} H_{j}(X)$. Now $A=A_{n-1} \otimes L_{n}$ is $A_{n-1}$-algebra isomorphic to $A_{n-1}[Y] /\left(H_{n}(Y)\right)$ by Lemma 1.3. Since $H_{n}(X)$ is irreducible in $A_{n-1}[Y]$ we conclude that $A$ is a field.

The trivial observation that 2) implies 1) completes the proof of the proposition.

Prop. 1.5 motivates the definition of the radical group which we present next. An element $[f]$ of $H^{2}(C, U(F))$ gives rise to a chain of fields $L_{0} \subseteq L_{1} \subseteq \cdots \subseteq L_{t-1}$ defined inductively in the following way. Let $L_{0}=F$. When $L_{i}$ has been defined, we then define $L_{i+1}$ to be a splitting field for the polynomial $h_{i+1}(X)$ over $L_{i}$, (see Remark 1.1).

We define $R_{f, i}$ for $1 \leq i \leq t$ to be the maximal subgroup of $E_{i}$ with the property that the image of $\left[f_{i}\right]$ under the natural map $H^{2}\left(E_{i}, U(F)\right) \rightarrow H^{2}\left(R_{f, i}, U\left(L_{i-1}\right)\right)$ is trivial.

Definition. The radical group $R_{f}$ of an element $[f]$ of $H^{2}(C, U(F))$ is defined to be the direct product $R_{f, 1} \times \cdots \times R_{f, t}$ where the $R_{f, i}$ are defined as above.

Observe that the definition of $R_{f}$ depends upon the order of the cyclic components $E_{i}$ of $C$. However, the non-triviality of $R_{f}$ shall be seen to be independent of the order of the cyclic components of $C$ (see Theorem 1.10). Once the order of the $E_{i}$ has been fixed, the definition of $R_{f}$ depends only upon the cohomology class of $f$.

It is convenient to make the following definition now.

Definition. Let the extension $S$ of $R$ have a separable residue class field extension, and let $[f]$ be an element of $H^{2}(G, U(S))$. Then the radical group $R_{f}$ of $[f]$ is defined to be the radical group of $[\bar{f}]$ where $[\bar{f}]$ is the image of $[f]$ under the natural maps

$$
H^{2}(G, U(S)) \rightarrow H^{2}(G, U(\bar{S})) \rightarrow H^{2}(C, U(\bar{S}))
$$

and $C$ is the center of the first ramification group of $S$ over $R$.

The following observation is immediate from the definition of the radical 
group, since the higher ramification groups of a tamely ramified extension vanish.

Proposition 1.6. If $S$ is a tamely ramified extension of $R$, then $R_{f}=(1)$ for each element $[f]$ of $H^{2}(G, U(S))$.

The following example shows that $R_{f}$ need not equal $C$.

ExAmple 1.7. Let $R=Z[X]_{(2)}$ and $S=R[\sqrt{2}]$. Then $S$ is a wildly ramified extension of $R$ and $C=(1, \sigma)$ is cyclic of order two. Let $f$ be the element of $Z^{2}(C, U(S))$ defined by $f(\sigma, \sigma)=X$. Then since $h(Y)=Y^{2}-X$ is irreducible over $\bar{S}=(Z /(2 Z))(X)$ we conclude that $R_{f}=(1)$.

The following proposition states necessary and sufficient conditions for the $i^{t h}$ component of the radical group to be trivial.

Proposition 1.8. The group $R_{f, i}$ is trivial if and only if $h_{i}(X)$ is irreducible in $L_{i-1}[X]$.

Proof. Let $h_{i}(X)=X^{e_{i}}-a_{i}=\left(X^{m_{i}}-b_{i}\right)^{e_{i} / m_{i}}$ be a factorization of $h_{i}(X)$ in $L_{i-1}[X]$ with $X^{m_{i}}-b_{i}$ irreducible. Note that $b_{i}^{e_{i} / m_{i}}=a_{i}$. We shall prove that $R_{f, i}=\left(\sigma^{m_{i}}\right)$ where $\sigma$ is a generator of the cyclic group $E_{i}$. To show that $\left(\sigma^{m_{i}}\right)$ is contained in $R_{f, i}$ we observe that $f_{i}$ is cohomologous to the trivial 2-cocycle. For the order of $\left(\sigma^{m_{i}}\right)$ is $e_{i} / m_{i}$ and $H^{2}\left(\left(\sigma^{m_{i}}\right), U\left(L_{i-1}\right)\right)=U\left(L_{i-1}\right) /\left[U\left(L_{i-1}\right)\right]^{e_{i} / m_{i}}$.

It remains to show that $R_{f, i}$ is contained in $\left(\sigma^{m_{i}}\right)$. Let $\sigma^{x}$ denote a generator of $R_{f, i}$. Then $a_{i}=c_{i}^{e_{i} / x}$ for some element $c_{i}$ in $U\left(L_{i-1}\right)$. From the inclusion $\left(\sigma^{m_{i}}\right) \subseteq R_{f, i}$ it follows that $e_{i} / m_{i}$ divides $e_{i} / x$ so that $\left(e_{i} / m_{i}\right) d=x$ for some positive integer $d$. The equalities $b_{i}^{e_{i} / m_{i}}=c_{i}^{e_{i} / x}=\left(c_{i}^{d}\right)^{e_{i} / m_{i}}$ imply that $b_{i}=c_{i}^{d}$. Therefore $X^{m_{i}}-b_{i}=\left(X^{x}-c_{i}\right)^{d}$. Since $X^{m_{i}}-b_{i}$ is irreducible over $L_{i-1}$ we conclude that $d=1$ and so $x=m_{i}$. Therefore $R_{f, i}$ is contained in $\left(\sigma^{m_{i}}\right)$.

The group $R_{f, i}$ is trivial therefore if and only if $m_{i}=e_{i}$, that is if and only if $h_{i}(X)$ is irreducible in $L_{i-1}[X]$.

Proposition 1.9. The radical group $R_{f}$ is trivial if and only if each polynomial $h_{i}(X)$ is irreducible in a splitting field for $\prod_{j<i} h_{j}(X)$ over $F$.

Proof. The radical group $R_{f}$ is trivial if and only if each cyclic component $R_{f, i}$ is trivial. By Prop. 1.7, the group $R_{f, i}$ is trivial if and only if $h_{i}(X)$ is irreducible in $L_{i-1}[X]$. However, the field $L_{i-1}$ was defined to be a splitting 
field for $\prod_{j<i} h_{j}(X)$ over $F$.

Now. we may prove the main theorem of this section.

THEOREM 1.10. The following statements are equivalent

1) the crossed product $\Delta(f, F, C)$ is semi-simple

2) $\Delta(f, F, C)$ is a field

3) the radical group $R_{f}$ is trivial

Proof. By Prop. 1.2, the crossed product $\Delta(f, F, C)$ is $F$-algebra isomorphic to the tensor product $A_{1} \otimes \cdots \otimes A_{t}$ over $F$ where $A_{i}=F[X] /\left(h_{i}(X)\right)$. Combining the results of Prop. 1.5 and Prop. 1.9 we arrive at the desired equivalence.

The following corollary gives technical information about the radical of $\Delta(f, F, C)$ in the case when $\Delta(f, F, C)$ is not a field which shall be of use in Section 2. Let $C=E_{1} \times \cdots \times E_{t}$ be a decomposition of the Abelian $p$-group $C$ into a direct product of cyclic groups; and for convenience of notation let $E_{0}=(1)$.

CoRollary 1.11. If the crossed product $\Delta(f, F, C)$ is not a field, then there exists an element of the form $u_{\tau}-\delta$ in rad $\Delta(f, F, C)$ where $\tau \neq 1$ is in $E_{q}$ for some $q \geq 1$, and $\delta$ is in the subring $\Delta\left(f, F, E_{1} \times \cdots \times E_{q-1}\right)$.

Proof. We assume as usual that the 2-cocycle $f$ has been normalized in the sense of Abelian p-groups. The assumption that $\Delta(f, F, C)$ is not a field implies that the radical group $R_{f}$ of $[f]$ is non-trivial according to the theorem. We may therefore consider the least positive integer $q$ for which the component $R_{f, q}$ is non-trivial. By the choice of $q$ it is clear that the crossed product $\Delta\left(f, F, E_{1} \times \cdots \times E_{q-1}\right)$ is a field which shall henceforth be denoted by $L$. Let $\sigma$ denote a generator of $E_{q}$. Then the $L$-algebra map $\psi: \Delta\left(f, F, E_{1} \times \cdots \times E_{q}\right)$ $\rightarrow L[X] /\left(h_{q}(X)\right)$ induced by defining $\psi\left(u_{\sigma}\right)=X$ is an $L$-algebra isomorphism. If $h_{q}(X)=\left(X^{m}-b\right)^{e / m}$ is a factorization of $h_{q}(X)$ in $L[X]$ with $X^{m}-b$ irreducible, then the fact that $L[X] /\left(h_{q}(X)\right)$ is not a field implies that $m<e$. The radical of $L[X] /\left(h_{q}(X)\right)$ is generated by the residue class of the element $X^{m}-b$, whose preimage in $\Delta\left(f, F, E_{1} \times \cdots \times E_{q}\right)$ under $\phi$ is of the form $u_{\tau}-\delta$ where $\tau=\sigma^{m}$ and $\delta$ is in $\Delta\left(f, F, E_{1} \times \cdots \times E_{q-1}\right)$. Note that $\tau \neq 1$ because $1 \leq m<e$. The fact that $\Delta(f, F, C)$ is a finitely generated commutative $\Delta\left(f, F, E_{1} \times \cdots \times E_{q}\right)$. algebra now implies that $u_{\tau}-\delta$ is in $\operatorname{rad} \Delta(f, F, C)$. 
2. p-groups. In Section 1 we noted that the crossed product $\Delta(f, S, G)$ is a $\Pi$-principal hereditary order if and only if $\Delta(\bar{f}, \bar{S}, G)$ is a semi-simple ring. The purpose of this section is to establish that $\Delta\left(\bar{f}, \bar{S}, G_{1}\right)$ is semi-simple if and only if $\Delta(\bar{f}, \bar{S}, C)$ is semi-simple, where $C$ denotes the center of the first ramification group $G_{1}$. Our object of study in this section is therefore the crossed product $\Delta\left(f, F, G_{1}\right)$ where $G_{1}$ is a $p$-group with trivial action on a field $F$ of characteristic $p$.

The notion of a splitting field of a cohomology class shall play an important role in Sections 2 and 3 by reducing questions concerning semi-simplicity to the case of a trivial crossed product.

Definition. Let $G$ be a finite group, and $F$ and $K$ fields such that $K$ is a $G$-ring over $F$. Let $[f]$ be an element of $H^{2}(G, U(K))$. Then an extension field $L$ of $K$ is called $a$ splitting field of $[f]$ if $[f]$ is in the kernel of the natural map $H^{2}(G, U(K)) \rightarrow H^{2}(G, U(L))$ induced by the inclusion of $K$ in $L$. If $L$ is a splitting field for $[f]$ we say that $L$ is a splitting field of the crossed product $\Delta(f, K, G)$. Finally, if a splitting field $L$ of $\Delta(f, K, G)$ is a purely inseparable extension of $K$ we call $L$ a purely inseparable splitting field of $\Delta(f, K, G)$.

Lemma 2.1. Let $F$ be a field of characteristic $p \neq 0$, and $C$ an Abelian $p$-group with trivial action on $F$. Let $[f]$ be an element of $H^{2}(C, U(F))$. Then the crossed product $\Delta(f, F, C)$ has a purely inseparable splitting field $L$. In the case when $\Delta(f, F, C)$ is a field we may take $L=\Delta(f, F, C)$.

Proof. Let $C=E_{1} \times \cdots \times E_{t}$ be a decomposition of $C$ into a direct product of cyclic groups. We may assume that $f$ is normalized in the sense of Abelian $p$-groups, and write $f=f_{1} \cdots f_{t}$ where the element $f_{i}$ of $Z^{2}\left(E_{i}, U(F)\right)$ is normalized in the sense of cyclic groups. Let $a_{i}$ be an element of $U(F)$ such that $\left[f_{i}\right]$ corresponds to $a_{i} \bmod [U(F)]^{e_{i}}$ under the canonical identification $H^{2}\left(E_{i}, U(F)\right)$ $=U(F) /[U(F)]^{e_{i}}$ where $e_{i}$ denotes the order of $E_{i}$. Let $L$ be the field obtained by adjoining the roots of the polynomials $X^{e_{i}}-a_{i}$ to $F$. Then $L$ is a purely inseparable extension of $F$ and $[f]$ is in the kernel of the map $H^{2}(C, U(F)) \rightarrow$ $H^{2}(C, U(L))$ induced by the inclusion of $F$ in $L$.

In the case when $\Delta(f, F, C)$ is a field let $L=\Delta(f, F, C)$. To verify that $L$ is a splitting field of $\Delta(f, F, C)$ it is sufficient to observe that $X^{e_{i}}-a_{i}=\left(X-u_{o}\right)_{0}^{e_{i}}$ 
so that each polynomial $X^{e_{i}}-a_{i}$ splits into linear factors in $L[X]$. Since $L$ is a splitting field for the polynomial $\Pi_{i}\left(X^{e_{i}}-a_{i}\right)$ over $F$ it is clear that $L$ is a purely inseparable extension of $F$.

Let $G_{1}$ be a $p$-group with trivial action on a field $F$ of characteristic $p$. The main theorem of this section states that the crossed product $\Delta\left(f, F, G_{1}\right)$ is semi-simple if and only if $\Delta(f, F, C)$ is a field where $C$ is the center of $G_{1}$. The proof involves an inductive process.

Consider the following chain of subgroups of the $p$-group $G_{1}$

$$
C_{n} \supset \cdots \supset C_{i} \supset \cdots \supset C_{0} \supset C_{-1}
$$

where the groups $C_{i}$ are defined inductively in the following way. Let $C_{-1}=(1)$. When $C_{i}$ has been defined, we then define $C_{i+1}$ to be the preimage of $\overline{C_{i+1}}$ in $G_{1}$ where $\overline{C_{i+1}}$ is the center of $G_{1} / C_{i}$. Note that $C_{0}=C$ where $C$ is the center of $G_{1}$, and $C_{n}=G_{1}$. It is easy to verify that each $C_{i}$ is a normal subgroup of $G_{1}$. Furthermore, each inclusion $C_{i} \subset C_{i+1}$ is strict since $G_{1}$ is a $p$-group. The following lemma states a property of the subgroups $C_{i}$ which shall be useful later in this section.

Lemma 2.2. Let $\rho$ be an element of $C_{k} / C_{k-2}$ not in the subgroup $C_{k-1} / C_{k-2}$. Then there exists an element $\tau$ in $G_{1} / C_{k-2}$ such that the commutator $c=\tau \rho \tau^{-1} \rho^{-1}$ is in $C_{k-1} / C_{k-2}$ and $c \neq 1$.

Proof. Suppose that $\tau \rho=\rho \tau$ for all elements $\tau$ in $G_{1} / C_{k-2}$. Since $C_{k-1} / C_{k-2}$ is by definition the center of $G_{1} / C_{k-2}$ it would then follow that $\rho$ is in $C_{k-1} / C_{k-2}$ which contradicts the assumption on $\rho$. Therefore we may consider an element $\tau$ of $G_{1} / C_{k-2}$ such that $\tau \rho \neq \rho \tau$.

Now the isomorphism $\left(G_{1} / C_{k-2}\right) /\left(C_{k-1} / C_{k-2}\right) \approx G_{1} / C_{k-1}$ together with the fact that $C_{k} / C_{k-1}$ is the center of $G_{1} / C_{k-1}$ implies that $\tau$ commutes with $\rho$ modulo $C_{k-1} / C_{k-2}$. Therefore $\tau \rho=c \rho \tau$ for some element $c$ in $C_{k-1} / C_{k-2}$ with $c \neq 1$.

The lemma concerning the existence of purely inseparable splitting fields shall be used to prove the next proposition.

Proposition 2.3. Let $G_{1}$ be a p-group with trivial action on a field $F$ of characteristic $p$, and let $[f]$ be an element of $H^{2}\left(G_{1}, U(F)\right)$. Then there exists a chain of fields

$$
F=L_{0} \subset L_{1} \subset \cdots \subset L_{i} \subset \cdots \subset L_{n}
$$


and 2-cocycles $g_{i}$ in $Z^{2}\left(G_{1}, U\left(L_{i}\right)\right)$ such that

1) each extension $L_{i} \subset L_{i+1}$ is purely inseparable

2) $g_{i}$ is cohomologous to the image of $f$ in $Z^{2}\left(G_{1}, U\left(L_{i}\right)\right)$ for each $i$

3) each $g_{i}$ is in the image of the inflation map $Z^{2}\left(G_{1} / C_{i-1}, U\left(L_{i}\right)\right) \rightarrow Z^{2}\left(G_{1}, U\left(L_{i}\right)\right)$

Proof. The construction of the fields $L_{i}$ and the 2-cocycles $g_{i}$ is done inductively. Let $L_{0}=F$ and $g_{0}=f$. It is clear that $L_{0}$ and $g_{0}$ satisfy statements 1), 2), and 3). When $L_{i}$ and $g_{i}$ have been defined, we then define $L_{i+1}$ and $g_{i+1}$ in the following way.

For convenience of notation we denote the preimage of $g_{i}$ in $Z^{2}\left(G_{1} / C_{i-1}, U\left(L_{i}\right)\right)$ by $g_{i}$ also. Then the field $L_{i+1}$ is defined to be a purely inseparable splitting field for the crossed product $\Delta\left(g_{i}, L_{i}, C_{i} / C_{i-1}\right)$. The existence of such a field $L_{i+1}$ is guaranteed by Lemma 2.1 ; when $\Delta\left(g_{i}, L_{i}, C_{i} / C_{i-1}\right)$ is a field we take $L_{i+1}=\Delta\left(g_{i}, L_{i}, C_{i} / C_{i-1}\right)$.

We next use $L_{i+1}$ in order to define the 2-cocycle $g_{i+1}$. Let $\hat{g}_{i}$ denote the image of $g_{i}$ in $Z^{2}\left(G_{1}, U\left(L_{i+1}\right)\right)$ under the map of $Z^{2}\left(G_{1}, U\left(L_{i}\right)\right)$ into $Z^{2}\left(G_{1}, U\left(L_{i+1}\right)\right)$ induced by the inclusion of $L_{i}$ in $L_{i+1}$. From the definition of $L_{i+1}$ it follows that the preimage of $\left[\hat{g}_{i}\right]$ in $H^{2}\left(G_{1} / C_{i-1}, U\left(L_{i+1}\right)\right)$ is trivial on $C_{i} / C_{i-1} \times C_{i} / C_{i-1}$.

Consider the following diagram

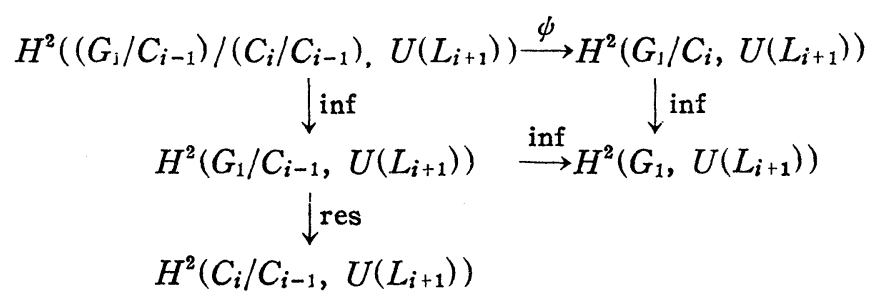

where the map $\psi$ is induced by the second Noether isomorphism theorem. It may be verified from the definitions of the maps that the diagram is commutative. Furthermore, by Prop. A. 7 we know that the column is exact. By diagram chasing we conclude that there exists a 2-cocycle $g_{i+1}$ in $Z^{2}\left(G_{1}, U\left(L_{i+1}\right)\right)$ cohomologous to $\hat{g}_{i}$ and in the image of the inflation map $Z^{2}\left(G_{1} / C_{i}, U\left(L_{i+1}\right)\right) \rightarrow$ $Z^{2}\left(G_{1}, U\left(L_{i+1}\right)\right)$. Observe that since the map $H^{2}\left(G_{1} / C_{i}, U\left(L_{i+1}\right)\right) \rightarrow H^{2}\left(G_{1}, U\left(L_{i+1}\right)\right)$ is an injection, we may assume that the preimage of $g_{i+1}$ in $Z^{2}\left(G_{1} / C_{i}, U\left(L_{i+1}\right)\right)$ is normalized on $C_{i+1} \times C_{i+1}$ in the sense of Abelian $p$-groups.

The notation used in the statement of Prop. 2.3 shall be in use throughout the rest of Section 2. The next object is to prove that each crossed product 
$\Delta\left(g_{i}, L_{i}, C_{i} / C_{i-1}\right)$ is a field whenever $\Delta(f, F, C)$ is a field. In order to do this we present three lemmas. The first two are of a general nature and shall be referred to several times in the paper. The third lemma gives technical information about the $g_{i}$ and $L_{i}$ of Prop. 2.3.

Lemma 2.4. Let the extension $S$ of $R$ be an extension of Artin rings. Then $(\mathrm{rad} S) \cap R$ is a nilpotent two-sided ideal of $R$. Therefore $(\mathrm{rad} S) \cap R$ is contained in rad $R$.

Proof. Since $S$ is an Artin ring it is well known that $\operatorname{rad} S$ is a nilpotent two-sided ideal of $S$. It follows easily now that $(\operatorname{rad} S) \cap R$ is a nilpotent twosided ideal of $R$ and is therefore contained in $\operatorname{rad} R$.

Lemma 2.5. Let $G$ be a finite group, $R$ a unitary commutative ring, and $A$ a G-ring over $R$. Let $G=\cup H_{\tau_{i}}$ be a disjoint right coset decomposition of $G$ relative to the subgroup $H$ of $G$. If $[f]$ is an element of $H^{2}(G, U(A))$, then the crossed product $\Delta(f, A, G)$ is a free left $\Delta(f, A, H)$-module with free generators $\left\{u_{i i}\right\}$.

Proof. Clearly the set $\left\{u_{\tau_{i}}\right\}$ generates $\Delta(f, A, G)$ as a left $\Delta(f, A, H)$ module. In order to show that $\left\{u_{\tau_{i}}\right\}$ is a free basis we shall show that if $\delta=\sum \delta_{i} u_{\tau_{i}}=0$ with the $\delta_{i}$ in $\Delta(f, A, H)$, then $\delta_{i}=0$ for each $i$. Write $\delta_{i}=\sum_{h} a_{h}^{(i)} u_{h}$ where each $a_{h}^{(i)}$ is in $A$ and each $h$ is in $H$. Then $\delta=\sum_{i} \sum_{h} a_{h}^{(i)} f\left(h, \tau_{i}\right) u_{h \tau_{i}}$. The coefficient of $u_{h \tau_{i}}$ is therefore $a_{h}^{(i)} f\left(h, \tau_{i}\right)$. Since $\Delta(f, A, G)$ is a free left $A$-module with free generators $u_{\tau}$ for $\tau$ in $G$, and the $f\left(h, \tau_{i}\right)$ are in $U(A)$, we conclude that $a_{h}^{(i)}=0$ for each $h$ and $i$. Therefore $\delta_{i}=0$ for each $i$.

Denote the crossed product $\Delta\left(g_{k-1}, L_{k-1}, C_{k} / C_{k-2}\right)$ by $\Delta_{k}$ and the crossed product $\Delta\left(g_{k-1}, L_{k-1}, C_{k-1} / C_{k-2}\right)$ by $\Delta_{k-1}$. Observe that $\Delta_{k-1}$ is a subring of $\Delta_{k}$.

Lemma 2.6. The crossed products $\Delta_{k}$ and $\Delta_{k-1}$ satisfy the following rules

1) $u_{\tau}\left(\operatorname{rad} \Delta_{k}\right)\left(u_{-}\right)^{-1} \subset \operatorname{rad} \Delta_{k}$ for each $\tau$ in $G_{1} / C_{k-2}$

2) $\left(\operatorname{rad} \Delta_{k}\right) \cap \Delta_{k-1} \subset \operatorname{rad} \Delta_{k-1}$

3) $\Delta_{k-1}$ is contained in the center of $\Delta_{k}$

Proof. In order to prove statement 1) we first observe that $\operatorname{rad} \Delta_{k}$ is a nilpotent two-sided ideal of $\Delta_{k}$ since $\Delta_{k}$ is an Artin ring. Using the fact that $\operatorname{rad} \Delta_{k}$ is two-sided together with the fact that $C_{k} / C_{k-2}$ is a normal subgroup of $G_{1} / C_{k-2}$ we can conclude that $u_{\tau}\left(\operatorname{rad} \Delta_{k}\right)\left(u_{\tau}\right)^{-1}$ is a two-sided ideal of $\Delta_{k}$. 
And the nilpotency of $u_{\tau}\left(\operatorname{rad} \Delta_{k}\right)\left(u_{\tau}\right)^{-1}$ follows immediately from that of $\operatorname{rad} \Delta_{k}$. Therefore, since $u_{\tau}\left(\operatorname{rad} \Delta_{k}\right)\left(u_{\tau}\right)^{-1}$ is a nil ideal of $\Delta_{k}$ we know that it is contained in $\operatorname{rad} \Delta_{k}$.

Assertion 2) follows immediately from Lemma 2.4.

We shall make use of Prop. A. 1 to prove that $\Delta_{k-1}$ is contained in the center of $\Delta_{k}$. For let $\lambda=\sum a_{\mathrm{p}} u_{\mathrm{p}}$ denote any element of $\Delta_{k-1}$, where the elements $\rho$ are in $C_{k-1} / C_{k-2}$ and the $a_{\rho}$ are in $L_{k-1}$. Since $C_{k} / C_{k-2}$ acts trivially on $L_{k-1}$, we know that $\lambda$ is in the center of $\Delta_{k}$ if and only if $\lambda_{\tau}=u_{\tau} \lambda$ for each element $\tau$ of $C_{k} / C_{k-2}$. Now $\lambda u_{\tau}=\sum a_{\rho} g_{k-1}(\rho, \tau) u_{\rho \tau}$. On the other hand, $u_{\tau} \lambda=$ $\sum a_{\rho} g_{k-1}(\tau, \rho) u_{\tau p}=\sum a_{\rho} g_{k-1}(\rho, \tau) u_{\rho}$ since $C_{k-1} / C_{k-2}$ is in the center of $C_{k} / C_{k-2}$ and $g_{k-1}(\tau, \rho)=g_{k-1}(\rho, \tau)$ according to Prop. A. 1 . Therefore $\lambda u_{\tau}=u_{\tau} \lambda$ for every $\lambda$ in $\Delta_{k-1}$ and $\tau$ in $C_{k} / C_{k-2}$, and so $\Delta_{k-1}$ is contained in the center of $\Delta_{k}$.

It is in the next proposition that we make use of the fact that the extension $L_{i+1}$ of $L_{i}$ is purely inseparable for each $i$.

Proposition 2.7. If the crossed product $\Delta(f, F, C)$ is a field then each crossed product $\Delta\left(g_{i}, L_{i}, C_{i} / C_{i-1}\right)$ is a field.

Proof. The proof is by contradiction. Suppose therefore that not all the commutative rings $\Delta\left(g_{i}, L_{i}, C_{i} / C_{i-1}\right)$ are fields. By hypothesis, $\Delta\left(g_{0}, L_{0}, C_{0}\right)=$ $\Delta(f, F, C)$ is a field so we may consider the least positive integer $k$ such that $\Delta\left(g_{k}, L_{k}, C_{k} / C_{k-1}\right)$ is not a field. We shall show first that the assumption that $\Delta\left(g_{k}, L_{k}, C_{k} / C_{k-1}\right)$ is not semi-simple implies that $\Delta\left(g_{k-1}, L_{k-1}, C_{k} / C_{k-2}\right)$ is not semi-simple. Then we shall show that the semi-simplicity of $\Delta\left(g_{k-1}, L_{k-1}, C_{k-1} / C_{k-2}\right)$ implies the semi-simplicity of the crossed product $\Delta\left(g_{k-1}, L_{k-1}, C_{k} / C_{k-1}\right)$ and thus arrive at a contradiction.

We proceed to show that $\Delta\left(g_{k-1}, L_{k-1}, C_{k} / C_{k-2}\right)$ is not semi-simple. The first step is to establish a connection between $\Delta\left(g_{k-1}, L_{k-1}, C_{k} / C_{k-2}\right)$ and the commutative ring $\Delta\left(g_{k}, L_{k}, C_{k} / C_{k-1}\right)$. It follows from Prop. A. 7 that the sequence (1) $\rightarrow H^{2}\left(C_{k} / C_{k-2}, U\left(L_{k}\right)\right) \rightarrow H^{2}\left(C_{k}, U\left(L_{k}\right)\right)$ is exact. Since $g_{k}$ is cohomologous to $g_{k-1}$ in $Z^{2}\left(C_{k}, U\left(L_{k}\right)\right)$ we conclude therefore that their preimages are cohomologous in $Z^{2}\left(C_{k} / C_{k-2}, U\left(L_{k}\right)\right)$ by some map $\phi: C_{k} / C_{k-2} \rightarrow U\left(L_{k}\right)$. Then the map $\psi: \Delta\left(g_{k}, L_{k}, C_{k} / C_{k-2}\right) \rightarrow \Delta\left(g_{k-1}, L_{k}, C_{k} / C_{k-2}\right)$ induced by defining $\psi\left(a u_{\rho}\right)=a \phi(\rho) u_{\rho}$ for a in $L_{k}$ and $\rho$ in $C_{k} / C_{k-2}$ is an $L_{k}$-algebra isomorphism. The following diagram establishes the desired relation between the crossed 
products $\Delta\left(g_{k-1}, L_{k-1}, C_{k} / C_{k-2}\right)$ and $\Delta\left(g_{k}, L_{k}, C_{k} / C_{k-1}\right)$. Observe that the columns are exact.

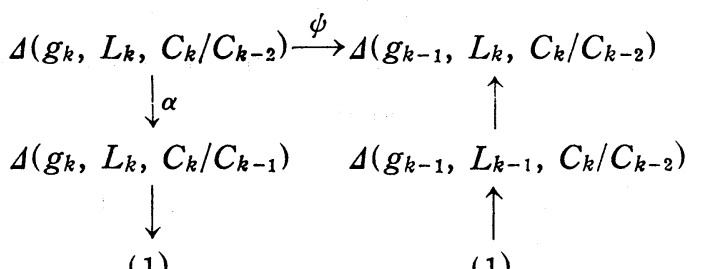

(1)

(1)

Explicitly, the map $\alpha$ is defined as follows. Let $N$ be the left ideal of $\Delta\left(g_{k}, L_{k}, C_{k} / C_{k-2}\right)$ generated by the set of all elements of the form $1-u_{\mathrm{p}}$ for $\rho$ in $C_{k-1} / C_{k-2}$. The ideal $N$ is in fact two-sided, and the natural map $\Delta\left(g_{k}, L_{k}, C_{k} / C_{k-2}\right) / N \rightarrow \Delta\left(g_{k}, L_{k}, C_{k} / C_{k-1}\right)$ is an $L_{k}$-algebra isomorphism. Then $\boldsymbol{\alpha}$ is defined to be the composition of the natural maps

$$
\Delta\left(g_{k}, L_{k}, C_{k} / C_{k-2}\right) \rightarrow \Delta\left(g_{k}, L_{k}, C_{k} / C_{k-2}\right) / N \rightarrow \Delta\left(g_{k}, L_{k}, C_{k} / C_{k-1}\right) .
$$

Note that the preimage of $\operatorname{rad} \Delta\left(g_{k}, L_{k}, C_{k} / C_{k-1}\right)$ is contained in rad $\Delta\left(g_{k}, L_{k}, C_{k} / C_{k-2}\right)$ since $N$ is contained in $\operatorname{rad} \Delta\left(g_{k}, L_{k}, C_{k} / C_{k-2}\right)$.

Now let $C_{k} / C_{k-1}=E_{1} \times \cdots \times E_{t}$ be a decomposition of the Abelian $p$-group $C_{k} / C_{k-1}$ into a direct product of cyclic groups. The assumption that $\Delta\left(g_{k}, L_{k}, C_{k} / C_{k-1}\right)$ is not semi-simple implies by Cor. 1.11 that there exists an element of the form $u=-\bar{\delta}$ in $\operatorname{rad} \Delta\left(g_{k}, L_{k}, C_{k} / C_{k-1}\right)$ where $\bar{\tau}$ is an element different from $\overline{1}$ in $E_{q}$ for some $q$ satisfying $1 \leq q \leq t$ and $\bar{\delta}$ is in $\Delta\left(g_{k}, L_{k}\right.$, $\left.E_{1} \times \cdots \times E_{q-1}\right)$. We may therefore consider an element $u_{:}-\delta$ of $\mathrm{rad}$ $\Delta\left(g_{k}, L_{k}, C_{k} / C_{k-2}\right)$ in the preimage of $u_{\bar{\tau}}-\bar{\delta}$.

We now use the element $u_{\tau}-\delta$ to produce a non-zero element $x$ in the radical of $\Delta\left(g_{k-1}, L_{k-1}, C_{k} / C_{k-2}\right)$. Write $\psi(\delta)$ in the form $\psi(\delta)=\sum a_{\rho} u_{\rho}$ where each $\rho$ is in $C_{k} / C_{k-2}$ and the elements $a_{\rho}$ are in $L_{k}$. Now by the assumption on $k$ we have taken $L_{k}=\Delta\left(g_{k-1}, L_{k-1}, C_{k-1} / C_{k-2}\right)$, so we may consider the isomorphism

$$
\theta: L_{k} \rightarrow \Delta\left(g_{k-1}, L_{k-1}, C_{k-1} / C_{k-2}\right)
$$

of subfields of $\Delta\left(g_{k-1}, L_{k}, C_{k} / C_{k-2}\right)$ which leaves $L_{k-1}$ element-wise fixed. Define the element $x$ of $\Delta\left(g_{k-1}, L_{k-1}, C_{k} / C_{k-2}\right)$ by setting $x=\phi(\tau) u_{\tau}-\delta_{1}$ where $\delta_{1}=\sum \theta\left(a_{\rho}\right) u_{\rho}$.

The next step is to show that $x$ is in $\operatorname{rad} d\left(g_{k-1}, L_{k}, C_{k} / C_{k-2}\right)$; and to do this it suffices to show that the image $\overline{\psi^{-1}(x)}$ of $\psi^{-1}(x)$ in $\Delta\left(g_{k}, L_{k}, C_{k} / C_{k-1}\right)$ 
is in the radical. Since $\Delta\left(g_{k}, L_{k}, C_{k} / C_{k-1}\right)$ is a commutative Artin ring, $\bar{\psi}^{-1}(x)$ is in the radical if and only if $\overline{\psi^{-1}(x)}$ is nilpotent. In order to prove the nilpotency of $\overline{\psi^{-1}(x)}$ we prove first that $\bar{\delta}^{P}={\overline{\psi^{-1}\left(\delta_{1}\right)}}^{P}$ where $P$ is the degree of $L_{k}$ over $L_{k-1}$. (Since $L_{k}$ is purely inseparable over $L_{k-1}$ we have the inclusion $L_{k}^{p} \subset L_{k-1}$. $) \quad$ Now $\bar{\delta}=\overline{\psi^{-1}\left(\sum \overline{\left.a_{\rho} u_{p}\right)}\right.}$ so that

$$
\bar{\delta}^{P}=\sum \overline{\left[\psi^{-1}\left(a_{\rho}\right) \psi^{-1}\left(u_{\rho}\right)\right]^{P}}=\sum \overline{\psi^{-1}\left(a_{\rho}^{P}\right) \psi^{-1}\left(u_{\rho}^{P}\right)} .
$$

On the other hand, using the fact that $a_{\rho}^{P}$ is in $L_{k-1}$ and is therefore left fixed by $\theta$ we obtain the equalities

$$
\begin{aligned}
& \overline{\psi^{-1}\left(\delta_{1}\right)}{ }^{P}=\sum \overline{\psi^{-1}\left(\theta\left(a_{\rho}^{P}\right)\right) \psi^{-1}\left(u_{\rho}^{P}\right)}=\sum \overline{\psi^{-1}\left(a_{\rho}^{r}\right)} \overline{\psi^{-1}\left(u_{\rho}^{P}\right)} \\
& =\bar{\delta}^{P}
\end{aligned}
$$

Since $u_{\bar{\tau}}-\bar{\delta}$ is in the radical of an Artin ring, we have that $\left(u_{\bar{\tau}}-\bar{\delta}\right)^{N}=0$ for some positive integer $N$. It is easy now to verify that $\overline{\psi^{-1}(x)}$ is nilpotent. For $\overline{\psi^{-1}(x)}{ }^{P N}=\left[u_{\bar{\Sigma}}-\overline{\psi^{-1}\left(\delta_{1}\right)}\right]^{P N}=u_{\bar{\tau}}^{P^{M}}-\bar{\delta}^{P N}=\left[u_{\bar{\tau}}-\bar{\delta}\right]^{P N}=\overline{0}$. This concludes the proof of the assertion that $x$ is in $\operatorname{rad} \Delta\left(g_{k-1}, L_{k}, C_{k} / C_{k-2}\right)$.

Since $x$ is in $\left(\operatorname{rad} \Delta\left(g_{k-1}, L_{k}, C_{k} / C_{k-2}\right)\right) \cap \Delta\left(g_{k-1}, L_{k-1}, C_{k} / C_{k-2}\right)$ we conclude from Lemma 2.4 that $x$ is in $\operatorname{rad} \Delta\left(g_{k-1}, L_{k-1}, C_{k} / C_{k-2}\right)$.

It remains to show that $x$ is non-zero. It may be observed from the first part of the proof that $\tau \neq \rho \bmod C_{k-1} / C_{k-2}$ for any element $\rho$ in the expression $\delta=\psi^{-1}\left(\sum a_{\rho} u_{\rho}\right)$, from which it certainly follows that $\tau \neq \rho$ for any such $\rho$. Since the crossed product $\Delta\left(g_{k-1}, L_{k-1}, C_{k} / C_{k-2}\right)$ is a free left $L_{k-1}$-module with free generators $u_{\sigma}$ for $\sigma$ in $C_{k} / C_{k-2}$ we conclude that $x \neq 0$. Therefore $\Delta\left(g_{k-1}, L_{k-1}\right.$, $\left.C_{k} / C_{k-2}\right)$ is not semi-simple, and this concludes the first part of the proof.

The rest of the proof involves showing that the semi-simplicity of $\Delta\left(g_{k-1}\right.$, $\left.L_{k-1}, C_{k-1} / C_{k-2}\right)$ implies the semi-simplicity of $\Delta\left(g_{k-1}, L_{k-1}, C_{k} / C_{k-2}\right) . \quad$ As in Lemma 2.6 we use the notation $\Delta_{k-1}=\Delta\left(g_{k-1}, L_{k-1}, C_{k-1} / C_{k-2}\right)$ and $\Delta_{k}=\Delta\left(g_{k-1}\right.$, $\left.L_{k-1}, C_{k} / C_{k-2}\right)$. Let $C_{k} / C_{k-2}=\bigcup_{i}\left(C_{k-1} / C_{k-2}\right) \rho_{i}$ be a disjoint right coset decomposition of $C_{k} / C_{k-2}$ relative to the subgroup $C_{k-1} / C_{k-2}$. Then an element $\delta$ of $\Delta_{k}$ can be written uniquely in the form $\delta=\sum_{i=1}^{t(\delta)} \delta_{i} u_{\rho_{i}}$ where each $\delta_{i}$ is in $\Delta_{k-1}$. We may assume that $\rho_{1}=1$.

The proof that $\Delta_{k}$ is semi-simple is by induction on $t(\delta)$. If $t(\delta)=1$, then $\delta$ is in $\left(\operatorname{rad} \Delta_{k}\right) \cap \Delta_{k-1}$, so that $\delta$ is in $\operatorname{rad} \Delta_{k-1}$ by Lemma 2.6. Since $\Delta_{k-1}$ is semi-simple we conclude that $\delta=0$. Now let $\delta=\sum_{i=1}^{t} \delta_{i} u_{\rho_{i}}$ be an element of $\operatorname{rad} \Delta_{k}$, 
with $\delta_{t} \neq 0$ and $\rho_{l}=1$. The induction hypothesis states that if $\gamma$ is an element of $\operatorname{rad} \iota_{k}$ and $t(\gamma)<t$, then $\gamma=0$. Consider the element $\rho_{t}$ of $C_{k} / C_{k-2}$. By Lemma 2.2 there exists an element $\tau$ in $G_{1} / C_{k-2}$ such that $\tau \rho_{t} \tau^{-1} \rho_{t}^{-1}=c_{t}$ is in $C_{k-1} / C_{k-2}$ and $c_{t} \neq 1$. For $1 \leq i \leq t-1$, let $c_{i}$ be defined by $c_{i}=\tau \rho_{i} \tau^{-1} \rho_{i}^{-1}$ and observe that each $c_{i}$ is in $C_{k-1} / C_{k-2}$.

Now form the element $\gamma=\delta-u_{\tau} \delta\left(u_{\tau}\right)^{-1}$. By Lemma 2.6 it follows that $\gamma$ is in $\mathrm{rad} \Delta_{k}$. Using the fact that $\Delta_{k-1}$ is contained in the center of $\Delta_{k}$ together with the definition of the $c_{i}$ one may obtain the equalities

$$
\begin{aligned}
r & =\sum_{i=1}^{t} \delta_{i} u_{\rho_{i}}-u_{\tau}\left(\sum_{i=1}^{t} \delta_{i} u_{\rho_{i}}\right)\left(u_{\tau}\right)^{-1} \\
& =\sum_{i=1}^{t} \delta_{i}\left[1-\frac{g_{k-1}\left(\tau, \rho_{i}\right) g_{k-1}\left(\tau \rho_{i}, \tau^{-1}\right)}{g_{k-1}\left(\tau, \tau^{-1}\right)} u_{c_{i}-1}\left(c_{i}, \rho_{i}\right)\right.
\end{aligned}
$$

For convenience of notation, let $\lambda_{i}=1-\frac{g_{k-1}\left(\tau, \rho_{i}\right) g_{k-1}\left(\tau \rho_{i}, \tau^{-1}\right)}{g_{k-1}\left(\tau, \tau^{-1}\right) g_{k-1}\left(c_{i}, \rho_{i}\right)} u_{c_{i}}$ for $1 \leq i \leq t$, and note that each $\lambda_{i}$ is in $\Delta_{k-1}$. It is easy to check that $c_{1}=1$ and $\lambda_{1}=0$ since $\rho_{1}=1$. Therefore $\gamma=\sum_{i=2}^{t}\left(\delta_{i} \lambda_{i}\right) u_{p i}$ with $\delta_{i} \lambda_{i}$ in $\Delta_{k-1}$ for $2 \leq i \leq t$. Hence $r$ is an element of $\operatorname{rad} \Delta_{k}$ such that $t(\gamma)<t$. By the induction hypothesis we may conclude that $\gamma=0$, and so $\delta_{i} \lambda_{i}=0$ for $2 \leq i \leq t$. But $\lambda_{t} \neq 0$ since $c_{t} \neq 1$, so that $\delta_{t}=0$ because $\Delta_{k-1}$ is a field. This contradicts the assumption that $\delta_{t} \neq 0$. Therefore $\operatorname{rad} \Delta_{k}=(0)$, and so $\Delta_{k}$ is semi-simple.

Lemma 2.8. If the crossed product $\Delta\left(g_{i}, L_{i}, C_{i} / C_{i-1}\right)$ is a field for some $i$, then the radical of $\Delta\left(g_{i}, L_{i}, C_{i}\right)$ is generated as a right ideal by the radical of $\Delta\left(1, L_{i}, C_{i-1}\right)$.

Proof. Recall that $g_{i}=1$ on $C_{i-1} \times C_{i-1}$. Let $N$ denote the right ideal of the trivial crossed product $\Delta\left(1, L_{i}, C_{i-1}\right)$ generated by the set of all elements of the form $1-u_{\sigma}$ with $\sigma$ in $C_{i-1}$. It follows at once from the exercise on p. 435 of [9] that $N$ is the radical of $\Delta\left(1, L_{i}, C_{i-1}\right)$. Therefore Lemma 1.4 now implies that $N \Delta\left(g_{i}, L_{i}, C_{i}\right)$ is contained in $\operatorname{rad} \Delta\left(g_{i}, L_{i}, C_{i}\right)$. In order to conclude that $N \Delta\left(g_{i}, L_{i}, C_{i}\right)$ is the radical of $\Delta\left(g_{i}, L_{i}, C_{i}\right)$, observe that the factor ring $\Delta\left(g_{i}, L_{i}, C_{i}\right) / N \Delta\left(g_{i}, L_{i}, C_{i}\right)$ is isomorphic to the crossed product $\Delta\left(g_{i}, L_{i}, C_{i} / C_{i-1}\right)$ and is therefore simple by hypothesis.

Proposition 2.9. Let $G_{1}$ be a p-group with trivial action on a field $F$ of characteristic $p$. Then the crossed product $\Delta\left(f, F, G_{1}\right)$ is semi-simple if and only if $\Delta(f, F, C)$ is a field where $C$ denotes the center of $G_{1}$. 
Proof. If $\Delta\left(f, F, G_{1}\right)$ is semi-simple, the fact that $\operatorname{rad} \Delta(f, F, C)$ is contained in $\operatorname{rad} \Delta\left(f, F, G_{1}\right)$ (see Lemma 1.4) implies that $\Delta(f, F, C)$ is also semi-simple. Therefore $\Delta(f, F, C)$ is a field according to Theorem 1.10 .

To prove the assertion in the other direction recall first of all that the assumption that $\Delta(f, F, C)$ is a field implies that each crossed product $\Delta\left(g_{i}, L_{i}, C_{i} / C_{i-1}\right)$ is a field by Prop. 2.7. We shall use this fact to prove inductively that $\Delta\left(f, F, C_{i}\right)$ is semi-simple for $0 \leq i \leq n$. Note that $\Delta\left(f, F, C_{0}\right)$ is semi-simple by hypothesis. So suppose that $\Delta\left(f, F, C_{i-1}\right)$ is semi-simple. In order to prove that $\Delta\left(f, F, C_{i}\right)$ is semi-simple consider the sequence of maps

$$
\Delta\left(f, F, C_{i}\right) \longrightarrow \Delta\left(f, L_{i}, C_{i}\right) \stackrel{\varphi}{\rightarrow} \Delta\left(g_{i}, L_{i}, C_{i}\right) \longrightarrow \Delta\left(g_{i}, L_{i}, C_{i} / C_{i-1}\right)
$$

where $\psi$ is the $L_{i}$-algebra isomorphism defined by $\psi\left(a u_{\tau}\right)=a \phi(\tau) u$ : for $a$ in $L_{i}$ and $\tau$ in $C_{i}$, and $\phi: C_{i} \rightarrow U\left(L_{i}\right)$ is the map by which $f$ is cohomologous to $g_{i}$ in $Z^{2}\left(C_{i}, U\left(L_{i}\right)\right)$. The other maps are the obvious ones.

Let $\delta$ denote any element of $\operatorname{rad} \Delta\left(f, F, C_{i}\right)$. We shall use the above sequence to prove that $\delta=0$. By applying Lemma 1.4 we may conclude that $\delta$ is in $\operatorname{rad} \Delta\left(f, L_{i}, C_{i}\right)$, so that $\psi(\delta)$ is in $\operatorname{rad} \Delta\left(g_{i}, L_{i}, C_{i}\right)$ since $\psi$ is an isomorphism. According to Lemma 2.8 the fact that $\Delta\left(g_{i}, L_{i}, C_{i} / C_{i-1}\right)$ is a field implies therefore that we may write $\psi(\delta)$ in the form $\phi(\delta)=\sum n_{i} \delta_{i}$ where each $n_{i}$ is in $\operatorname{rad} \Delta\left(1, L_{i}, C_{i-1}\right)$ and each $\delta_{i}$ is in $\Delta\left(g_{i}, L_{i}, C_{i}\right)$. Therefore $\delta=\sum \psi^{-1}\left(n_{i}\right) \psi^{-1}\left(\delta_{i}\right)$. From the definition of the isomorphism $\psi$, it follows that each element $\psi^{-1}\left(n_{i}\right)$ is in $\operatorname{rad} \Delta\left(f, L_{i}, C_{i-1}\right)$. Consider now a disjoint right coset decomposition $C_{i}=\bigcup_{j} C_{i-1} \rho_{j}$ of $C_{i}$ relative to the subgroup $C_{i-1}$. Then each element $\psi^{-1}\left(\delta_{i}\right)$ has a unique expression in the form $\psi^{-1}\left(\delta_{i}\right)=\sum_{j} \lambda_{j}^{(i)} u_{\rho j}$ where the $\lambda_{j}^{(i)}$ are in $\Delta\left(f, L_{i}, C_{i-1}\right)$. Therefore $\delta=\sum_{j}\left[\sum_{i} \psi^{-1}\left(n_{i}\right) \lambda_{j}^{(i)}\right] u_{\rho j}$. The fact that $\delta$ is in $\Delta\left(f, F, C_{i}\right)$ implies now that $\sum_{i} \psi^{-1}\left(n_{i}\right) \lambda_{j}^{(i)}$ is in $\Delta\left(f, F, C_{i-1}\right)$ for each $j$, so that $\sum_{i} \psi^{-1}\left(n_{i}\right) \lambda_{j}^{(i)}$ is in $\left(\operatorname{rad} \Delta\left(f, L_{i}, C_{i-1}\right)\right) \cap \Delta\left(f, F, C_{i-1}\right)$. It follows from Lemma 2.4 that $\left(\operatorname{rad} \Delta\left(f, L_{i}, C_{i-1}\right)\right) \cap \Delta\left(f, F, C_{i-1}\right)$ is contained in the radical of $\Delta\left(f, F, C_{i-1}\right)$. By the induction hypothesis, $\operatorname{rad} \Delta\left(f, F, C_{i-1}\right)=(0)$. Therefore $\sum \psi^{-1}\left(n_{i}\right) \lambda_{j}^{(i)}=0$ for each $j$, and so we conclude finally that $\delta=0$.

3. Hereditary orders. Let $S$ be the integral closure of a complete discrete rank one valuation ring $R$ in a finite Galois extension of the quotient field of $R$, and let $G$ denote the Galois group of the quotient field extension. Assume 
moreover that the residue class field extension $\bar{S}$ of $\bar{R}$ is separable. The purpose of this section is to prove the main theorem of the paper, namely that the crossed product $\Delta(f, S, G)$ is a $\Pi$-principal hereditary order if and only if the radical group $R_{f}$ is trivial. (See Section 1 for the definition of radical group.)

The results of Sections 1 and 2 together imply that $\Delta\left(\bar{f}, \bar{S}, G_{1}\right)$ is semisimple if and only if $R_{f}=(1)$, where $G_{1}$ denotes the first ramification group of $S$ over $R$. The crossed product $\Delta(f, S, G)$ is a $\Pi$-principal hereditary order if and only if $\Delta(\bar{f}, \bar{S}, G)$ is semi-simple. Therefore our next object is to prove that $\Delta(\bar{f}, \bar{S}, G)$ is semi-simple if and only if $\Delta\left(\bar{f}, \bar{S}, G_{1}\right)$ is semi-simple.

The first step is to reduce the problem to the inertial case. For the sake of completeness we prove the following proposition which has already been established by Harada in [10].

Proposition 3.1. Let $S$ be an integrally closed extension of a complete discrete rank one valuation ring $R$, and let $G_{0}$ denote the inertia group of $S$ over $R$. Let [f] denote an element of $H^{2}(G, U(S))$. Then the radical of the crossed product $\Delta(f, S, G)$ is generated as a right ideal by the radical of $\Delta\left(f, S, G_{0}\right)$.

Proof. Let $\Pi$ denote a prime element of $S$. Since $\Pi$ is in $\operatorname{rad} \Delta(f, S, G)$ and in $\operatorname{rad} \Delta\left(f, S, G_{0}\right)$ it suffices to prove that the radical of $\Delta(\bar{f}, \bar{S}, G)$ is generated as a right ideal by the radical of $\Delta\left(\bar{f}, \bar{S}, G_{0}\right)$. For convenience of notation we let $\bar{\Delta}=\Delta(\bar{f}, \bar{S}, G)$ and $\bar{\Delta}_{0}=\Delta\left(\bar{f}, \bar{S}, G_{0}\right)$.

Let $U$ denote the inertia ring of $S$ over $R$. Since $\bar{S}$ is a purely inseparable extension of $\bar{U}$, the inertia group $G_{0}$ acts trivially on $\bar{S}$. Furthermore, $\bar{U}=\bar{R}(\theta)$ for some element $\theta$ of $\bar{U}$ since $\bar{U}$ is a finite separable extension of $\bar{R}$.

Observe that the intersection $(\operatorname{rad} \bar{\Delta}) \cap \bar{\Delta}_{0}$ is contained in $\operatorname{rad} \bar{\Delta}_{0}$ under the natural injection of $\bar{\Delta}_{0}$ into $\bar{\Delta}$ according to Lemma 2.4 .

Now we may prove the proposition. Let $G=\cup G_{0} \tau_{i}$ be a disjoint right coset decomposition of $G$ relative to the normal subgroup $G_{0}$. Let $\delta$ be an element of $\operatorname{rad} \bar{\Delta}$ and write $\delta=\sum_{i=1}^{t(\delta)} \delta_{i} u_{\tau_{i}}$ where $\delta_{i}=\sum c_{h}^{(i)} u_{h}$ with the $h$ in $G_{0}$ and the $c_{h}^{(i)}$ in $U(\bar{S})$. Note that the elements $\delta_{i}$ are unique by Lemma 2.5. We shall prove by induction on $t(\delta)$ that each $\delta_{i}$ is in $\operatorname{rad} \bar{\Delta}_{0}$. For suppose that $t(\delta)=1$. Then $\delta=\delta_{i} u_{\tau_{i}}$ where $\delta_{i}$ is in $\bar{\Delta}_{0}$. The element $\delta\left(u_{\tau_{i}}\right)^{-1}$ is therefore in $(\operatorname{rad} \bar{\Delta}) \cap \bar{\Delta}_{0}$. By the above observation we conclude that $\delta_{i}$ is in $\operatorname{rad} \Delta_{0}$.

Now let $\delta=\sum_{i=1} \delta_{i} u_{\tau_{i}}$ be an element of $\operatorname{rad} \bar{\Delta}$ for which $t(\delta)=t$. The induction 
hypothesis states that if $t(\gamma)<t$ for an element $\gamma$ of $\operatorname{rad} \bar{\Delta}$ then each $\gamma_{i}$ is in $\operatorname{rad} \bar{\Delta}_{0}$ where $\gamma=\sum r_{i} u_{\tau_{i}}$. Consider the element $\alpha=\theta \delta-\delta \tau_{t}^{-1}(\theta)=\sum_{i=1}^{t-1}\left(\theta-\tau_{i} \tau_{t}^{-1}(\theta)\right) \delta_{i} u_{\tau_{i}}$. Since $\alpha$ is in $\operatorname{rad} \vec{\Delta}$ and $t(\alpha)<t$ it follows from the induction bypothesis that $\left(\theta-\tau_{i} \tau_{t}^{-1}(\theta)\right) \delta_{i}$ is in $\mathrm{rad} \bar{\Delta}_{0}$ for each $i$ such that $1 \leq i \leq t-1$. Since $G / G_{0}$ is the Galois group of $\bar{U}$ over $\bar{R}$ (see p. 32 of [5]) we have that $\tau_{i} \tau_{t}^{-1}(\theta)=\theta$ if and only if $i=t$. Therefore $\delta_{i}$ is in $\operatorname{rad} \bar{\Delta}_{0}$ for $1 \leq i \leq t-1$. Finally we observe that $\delta_{t} u_{\tau_{t}}$ is in $\operatorname{rad} \bar{\Delta}$ so that $\delta_{t}=\delta_{t} u_{\tau_{l}}\left(u_{\tau_{t}}\right)^{-1}$ is in $(\operatorname{rad} \bar{\Delta}) \cap \bar{\Delta}_{0}$ and hence in $\operatorname{rad} \bar{\Delta}_{0}$. Therefore $\delta_{i}$ is in $\operatorname{rad} \bar{\Delta}_{0}$ for $1 \leq i \leq t$ and this concludes the proof.

As in Section 2 we shall use the notion of a splitting field of a crossed product to reduce computations to the case of a trivial crossed product.

Proposition 3.2. Let $f$ be an element of $Z^{2}\left(G_{0}, U(S)\right)$. Then there exists a finite purely inseparable extension $L$ of $\bar{S}$ and a 2-cocycle $g$ of $Z^{2}\left(G_{0}, U(L)\right)$ such that $g$ is in the image of the inflation map $Z^{2}\left(G_{0} / G_{1}, U(L)\right) \rightarrow Z^{2}\left(G_{0}, U(L)\right)$ and is cohomologous to the image of $\bar{f}$ in $Z^{2}\left(G_{0}, U(L)\right)$.

Proof. The proof is by induction on the number of ramification groups. Let

$$
G_{\alpha(0)} \supset G_{\alpha(1)} \supset \cdots \supset G_{\alpha(n)} \supset G_{\alpha(n+1)}=(1)
$$

be the sequence of (distinct) ramification groups of the extension $S$ of $R$, observing that $\alpha(0)=0$ and $\alpha(1)=1$. We first construct a chain of fields $\bar{S}=L_{0} \subset L_{1} \subset \cdots \subset L_{n}$ and 2-cocycles $g_{i}$ of $Z^{2}\left(G_{0}, U\left(L_{i}\right)\right)$ such that each $L_{i+1}$ is a purely inseparable extension of $L_{i}$, and each $g_{i}$ is in the image of the inflation map $Z^{2}\left(G_{0} / G_{\alpha(n+1-i)}, U\left(L_{i}\right)\right) \rightarrow Z^{2}\left(G_{0}, U\left(L_{i}\right)\right)$ and is cohomologous to the image of $\bar{f}$ in $Z^{2}\left(G_{0}, U\left(L_{i}\right)\right)$.

We define $L_{0}=\bar{S}$ and $g_{0}=\bar{f}$. It is a trivial observation that $L_{0}$ and $g_{0}$ have the desired properties. When $L_{i}$ and $g_{i}$ have been defined, we then define $L_{i+1}$ and $g_{i+1}$ in the following way. For convenience of notation we denote the preimage of $g_{i}$ in $Z^{2}\left(G_{0} / G_{\alpha(n+1-i)}, U\left(L_{i}\right)\right)$ by $g_{i}$ also. Then $L_{i+1}$ is defined to be a finite purely inseparable splitting field for the crossed product $\Delta\left(g_{i}, L_{i}, G_{\alpha(n-i)} / G_{\alpha(n+1-i)}\right)$. The existence of such a field $L_{i+1}$ is guaranteed by Lemma 2.i, since $G_{\alpha(n-i)} / G_{\alpha(n+1-i)}$ is an Abelian $p$-group with trivial action on $L_{i}$. By an argument entirely similar to that used in the proof of Prop. 2.3 we may conclude the existence of a 2-cocycle $g_{i+1}$ in $Z^{2}\left(G_{0}, U\left(L_{i+1}\right)\right)$ which is in the image of the inflation map $Z^{2}\left(G_{0} / G_{\alpha(n-i)}, U\left(L_{i+1}\right)\right) \rightarrow Z^{2}\left(G_{0}, U\left(L_{i+1}\right)\right)$ and 
is cohomologous to the image of $\bar{f}$ in $Z^{2}\left(G_{0}, U\left(L_{i+1}\right)\right)$. We may prove the proposition now by taking $L=L_{n}$ and $g=g_{n}$.

The notation established in Prop. 3.2 shall be used throughout the rest of Section 3.

Proposition 3.3. The radical of the crossed product $\Delta\left(g, L, G_{0}\right)$ is generated as a right ideal by the radical of $\Delta\left(1, L, G_{1}\right)$.

Proof. Let $N$ denote the right ideal of $\Delta\left(1, L, G_{1}\right)$ generated by the set of all elements of the form $1-u_{\sigma}$ with $\sigma$ in $G_{1}$. Since $G_{1}$ is a $p$-group and $L$ has characteristic $p$, it follows at once from the exercise on p. 435 of [9] that $N$ is the radical of the trivial crossed product $\Delta\left(1, L, G_{1}\right)$.

It remains to show that $N \Delta\left(g, L, G_{0}\right)$ is the radical of $\Delta\left(g, L, G_{0}\right)$. Using the fact that $g$ is in the image of the inflation map $Z^{2}\left(G_{0} / G_{1}, U(L)\right) \rightarrow Z^{2}\left(G_{0}, U(L)\right)$ together with the fact that $G_{1}$ is a normal subgroup of $G_{0}$, one may conclude from the definition of $N$ that the right ideal $N \Delta\left(g, L, G_{0}\right)$ is equal to the left ideal $\Delta\left(g, L, G_{0}\right) N$. Lemma 1.4 now implies that $N \Delta\left(g, L, G_{0}\right)$ is contained in $\operatorname{rad} \Delta\left(g, L, G_{0}\right)$. To prove that $N \Delta\left(g, L, G_{0}\right)$ is the radical of $\Delta\left(g, L, G_{0}\right)$ it suffices therefore to show that the factor ring $\Delta\left(g, L, G_{0}\right) / N \Delta\left(g, L, G_{0}\right)$ is semi-simple. Now $\Delta\left(g, L, G_{0}\right) / N \Delta\left(g, L, G_{0}\right)$ is isomorphic to the crossed product $\Delta\left(g, L, G_{0} / G_{1}\right)$ in a natural way. Since $G_{0} / G_{1}$ acts trivially on $L$, and the order of $G_{0} / G_{1}$ is relatively prime to the characteristic of $L$, it follows from Theorem 1.1 of [7] that $\Delta\left(g, L, G_{0} / G_{1}\right)$ is $L$-separable and therefore semi-simple.

Proposition 3.4. The radical of the crossed product $\Delta\left(\bar{f}, \bar{S}, G_{0}\right)$ is generated as a right ideal by the radical of $\Delta\left(\bar{f}, \bar{S}, G_{1}\right)$.

Proof. The first step is to prove that the radical of $\Delta\left(\bar{f}, L, G_{0}\right)$ is generated as a right ideal by the radical of $\Delta\left(\bar{f}, L, G_{1}\right)$. Consider the 2-cocycle $g$ of $Z^{2}\left(G_{0}, U(L)\right)$ whose existence is established by Prop. 3.2, and let $\phi: G_{0} \rightarrow U(L)$ be the map by which $\bar{f}$ is cohomologous to $g$ in $Z^{2}\left(G_{0}, U(L)\right)$. It is well known that the map $\psi: \Delta\left(\bar{f}, L, G_{0}\right) \rightarrow \Delta\left(g, L, G_{0}\right)$ defined by $\psi\left(a u_{\tau}\right)=a \phi(\tau) u_{\tau}$ for $a$ in $L$ and $\tau$ in $G_{0}$ is an $L$-algebra isomorphism. The radical of $\Delta\left(g, L, G_{0}\right)$ is generated as a right ideal by the radical of $\Delta\left(1, L, G_{1}\right)$ according to Prop. 3.3. Since $\psi^{-1}\left[\Delta\left(g, L, G_{1}\right)\right]=\Delta\left(\bar{f}, L, G_{1}\right)$ we may conclude therefore that the radical of $\Delta\left(\bar{f}, L, G_{0}\right)$ is generated as a right ideal by the radical of $\Delta\left(\bar{f}, L, G_{1}\right)$.

Now consider an element $\delta$ of $\operatorname{rad} \Delta\left(\bar{f}, \bar{S}, G_{0}\right)$. It follows easily from 
Lemma 1.4 that $\delta$ is also in $\operatorname{rad} \Delta\left(\vec{f}, L, G_{0}\right)$, so that according to the first part of the proof we may write $\delta=\sum n_{i} \delta_{i}$ where each $n_{i}$ is in $\operatorname{rad} \Delta(\bar{f}, L, G)$ and the $\delta_{i}$ are in $\Delta\left(\bar{f}, L, G_{0}\right)$. Each element $\delta_{i}$ has a unique expression in the form $\delta_{i}=\sum \lambda_{j}^{(i)} u_{\rho_{j}}$ with the $\lambda_{j}^{(i)}$ in $\Delta\left(\bar{f}, L, G_{1}\right)$, where $G_{0}=\cup G_{1} \rho_{j}$ is a disjoint right coset decomposition of $G_{0}$ relative to the subgroup $G_{1}$. Therefore $\delta=\sum_{j}\left[\sum_{i} n_{i} \lambda_{j}^{(i)}\right] u_{\mathrm{p} j}$. Since $\delta$ is in $\Delta\left(\bar{f}, \bar{S}, G_{0}\right)$, the fact that $\Delta\left(\bar{f}, L, G_{0}\right)$ is a free left $\Delta\left(\bar{f}, L, G_{1}\right)$ module with free basis $\left\{u_{p i}\right\}$ implies that $\sum_{i} n_{i} \lambda_{j}^{(i)}$ is in $\Delta\left(\bar{f}, \bar{S}, G_{1}\right)$ for each $j$. Therefore each $\sum_{i} n_{i} \lambda_{j}^{(i)}$ is in $\left(\operatorname{rad} \Delta\left(\bar{f}, L, G_{1}\right)\right) \cap \Delta\left(\bar{f}, \bar{S}, G_{1}\right)$, which according to Lemma 2.4 is contained in $\operatorname{rad} \Delta\left(\bar{f}, \bar{S}, G_{1}\right)$. The fact that an element $\delta$ of $\operatorname{rad} \Delta\left(\bar{f}, \bar{S}, G_{0}\right)$ may be written in the form $\delta=\sum_{j}\left[\sum_{i} n_{i} \lambda_{j}^{(i)}\right] u_{\rho j}$ with each $\sum_{i} n_{i} \lambda_{j}^{(i)}$ in $\operatorname{rad} \Delta\left(\bar{f}, \bar{S}, G_{1}\right)$ establishes the assertion of the proposition.

Using Prop. 3.4 together with the results of Sections 1 and 2 we may now prove the main theorem of the paper.

TheOREM 3.5. Let $S$ be an integrally closed extension of a complete discrete rank one valuation ring $R$ such that the residue class field extension is separable, and let $[f]$ be an element of $H^{2}(G, U(S))$. Then the crossed product $\Delta(f, S, G)$ is a II-principal hereditary order if and only if the radical group $R_{f}$ of $[f]$ is trivial.

Proof. The crossed product $\Delta(f, S, G)$ is a $I I$-principal hereditary order if and only if the crossed product $\Delta(\bar{f}, \bar{S}, G)$ is semi-simple. By Prop. 3.1 we know that $\Delta(\bar{f}, \bar{S}, G)$ is semi-simple if and only if $\Delta\left(\bar{f}, \bar{S}, G_{0}\right)$ is semi-simple where $G_{0}$ denotes the inertia group of $S$ over $R$. And the crossed product $\Delta\left(\bar{f}, \bar{S}, G_{0}\right)$ is semi-simple if and only if $\Delta\left(\bar{f}, \bar{S}, G_{1}\right)$ is semi-simple according to Prop. 3.4, where $G_{1}$ is the first ramification group of $S$ over $R$. Prop. 2.9 implies in turn that $\Delta\left(\bar{f}, \bar{S}, G_{1}\right)$ is semi-simple if and only if $\Delta(\bar{f}, \bar{S}, C)$ is a field where $C$ denotes the center of $G_{1}$. Finally, the fact that $\Delta(\bar{f}, \bar{S}, C)$ is a field if and only if $R_{f}$ is trivial (see Theorem 1.10) establishes the assertion of the theorem.

We obtain at once from Theorem 3.5 the following result which has already been proved by Harada (see Theorem 2 of [10]).

Corollary 3.6. Let $R$ be a complete discrete rank one valuation ring with perfect residue class field, and let $S$ denote an integrally closed extension of $R$. If $[f]$ is an element of $H^{2}(G, U(S))$, then the crossed product $\Delta(f, S, G)$ is a $\Pi$ - 
principal hereditary order if and only if $S$ is a tamely ramified extension of $R$.

Proof. In the case when $S$ is a tamely ramified extension of $R$ it was proved in [7] that $\Delta(f, S, G)$ is a $I I$-principal hereditary order.

We prove the assertion in the other direction by contradiction. So suppose that the extension $S$ of $R$ is not tamely ramified. Then the center $C$ of the first ramification group $G_{1}$ is non-trivial. Since $\bar{R}$ is perfect, $\bar{f}$ is cohomologous to 1 on $C \times C$ by Cor. A. 5. From the definition of the radical group it now follows that $R_{f}=C$.

Appendix. Cohomology. In this appendix to the paper we present several general facts concerning cohomology which have application to the study of crossed products.

Proposition A.1. Let $F$ be a field of characteristic $p \neq 0$, and $G$ a group which acts trivially on $F$. Suppose that $\sigma$ and $\tau$ are elements of $G$ such that $\sigma \tau=\tau \sigma$. If the order of $\tau$ is a $p^{\text {th }}$ power, then $f(\sigma, \tau)=f(\tau, \sigma)$ for every 2-cocycle $f$ in $Z^{2}(G, U(F))$.

Proof. Let $p^{t}$ denote the order of $\tau$. By the associativity property of $f$ we obtain the equalities

$$
\begin{aligned}
& f\left(\tau, \sigma \tau^{p^{t}-1}\right) f\left(\sigma, \tau^{p^{t}-1}\right)=f\left(\tau \sigma, \tau^{p^{t}-1}\right) f(\tau, \sigma) \\
& f\left(\tau, \tau^{p^{t}-1} \sigma\right) f\left(\tau^{p^{t}-1}, \sigma\right)=f\left(\tau, \tau^{p^{t}-1}\right) \\
& f\left(\sigma \tau, \tau^{p^{t}-1}\right) f(\sigma, \tau)=f\left(\tau, \tau^{p^{t}-1}\right)
\end{aligned}
$$

Combining the above equalities we obtain that

$$
f\left(\sigma, \tau^{p^{t}-1}\right) f(\sigma, \tau)=f\left(\tau^{p^{t}-1}, \sigma\right) f(\tau, \sigma) .
$$

We next obtain an expression for $f\left(\tau^{p^{t}-1}, \sigma\right)$. Write $f\left(\tau^{p^{t-i}}, \sigma\right)=f\left(\tau^{p^{t-i-1}} \tau, \sigma\right)$ for $1 \leq i \leq p^{t}-1$. By combining the equalities

$$
\begin{aligned}
& f\left(\tau^{p^{t}-i-1} \tau, \sigma\right) f\left(\tau^{p^{t}-i-1}, \tau\right)=f\left(\tau^{p^{t}-i-1}, \tau \sigma\right) f(\tau, \sigma) \\
& f\left(\tau^{p^{t}-i-1}, \sigma \tau\right) f(\sigma, \tau)=f\left(\tau^{p^{t}-i-1} \sigma, \tau\right) f\left(\tau^{p^{t}-i-1}, \sigma\right)
\end{aligned}
$$

we get that

$$
f\left(\tau^{p^{t-i}}, \sigma\right)=f(\tau, \sigma) f\left(\sigma \tau^{p^{t-i-1}}, \tau\right) f\left(\tau^{p^{t}-i-1}, \sigma\right) / f(\sigma, \tau) f\left(\tau^{p^{i-i-1}}, \tau\right)
$$

for $1 \leq i \leq p^{t}-1$. By repeated use of this equality it follows that

$$
f\left(\tau^{p^{2}-1}, \sigma\right)=[f(\tau, \sigma) / f(\sigma, \tau)]^{p^{t}-1} \prod_{i=2}^{p^{t}} f\left(\sigma \tau^{p^{t}-i}, \tau\right) / f\left(\tau^{p^{t}-i}, \tau\right) .
$$


On the other hand, we may write $f\left(\sigma, \tau^{p^{t-i}}\right)=f\left(\sigma, \tau^{p^{t}-i-1} \tau\right)$ and obtain an expression for $f\left(\sigma, \tau^{p^{t}-1}\right)$. The associativity property of $f$ implies that

$$
f\left(\sigma, \tau^{p^{i}-i}\right)=f\left(\sigma \tau^{p^{i}-i-1}, \tau\right) f\left(\sigma, \tau^{p^{t}-i-1}\right) / f\left(\tau^{p^{t}-i-i}, \tau\right) .
$$

By repeated use of this equality we obtain that

$$
f\left(\sigma, \tau^{p^{t}-1}\right)=\prod_{i=2}^{p^{t}} f\left(\sigma \tau^{p^{t-i}}, \tau\right) / f\left(\tau^{p^{t}-i}, \tau\right) .
$$

Now we may conclude that $f(\sigma, \tau)=f(\tau, \sigma)$. For by substituting the above expressions for $f\left(\tau^{p^{t}-1}, \sigma\right)$ and $f\left(\sigma, \tau^{p^{t}-1}\right)$ into the equality $f\left(\sigma, \tau^{p^{t}-1}\right) f(\sigma, \tau)=$ $f\left(\tau^{p^{t}-1}, \sigma\right) f(\tau, \sigma)$ we get that $[f(\sigma, \tau)]^{p^{t}}=[f(\tau, \sigma)]^{p^{l}}$. Since $F$ has characteristic $p$, we conclude that $f(\sigma, \tau)=f(\tau, \sigma)$.

Corollary A.2. Let $F$ be a field of characteristic $p \neq 0$, and let $E$ and $G_{p}$ be groups with $G_{p}$ a p-group. If $E$ and $G_{p}$ act trivially on $F$, then the natural map

$$
H^{2}\left(E \times G_{p}, U(F)\right) \rightarrow H^{2}(E, U(F)) \times H^{2}\left(G_{p}, U(F)\right)
$$

is an isomorphism.

Proof. Define a map

$$
\varphi: Z^{2}\left(E \times G_{p}, U(F)\right) \rightarrow Z^{2}(E, U(F)) \times Z^{2}\left(G_{p}, U(F)\right)
$$

by $\varphi(f)=f_{1} f_{2}$ where $f_{1}$ is the restriction of $f$ to $E \times E$ and $f_{2}$ is the restriction of $f$ to $G_{p} \times G_{p}$. Then $\varphi$ induces a well-defined map

$$
\bar{\varphi}: H^{2}\left(E \times G_{p}, U(F)\right) \rightarrow H^{2}(E, U(F)) \times H^{2}\left(G_{p}, U(F)\right) .
$$

We shall show that the map $\bar{\varphi}$ is a group isomorphism.

It follows from the definition of $\varphi$ that $\bar{\varphi}$ is a homomorphism of groups. We next observe that $\varphi$ is an epimorphism. For let $f_{1} f_{2}$ be any element of $Z^{2}(E, U(F)) \times Z^{2}\left(G_{p}, U(F)\right)$. Then define the map $f:\left(E \times G_{p}\right) \times\left(E \times G_{p}\right) \rightarrow U(F)$ by $f\left(\sigma_{1} \tau_{1}, \sigma_{2} \tau_{2}\right)=f_{1}\left(\sigma_{1}, \sigma_{2}\right) f_{2}\left(\tau_{1}, \tau_{2}\right)$ where $\sigma_{1}$ and $\sigma_{2}$ are in $E$ and $\tau_{1}$ and $\tau_{2}$ are in $G_{p}$. It is easy to verify that $f$ is an element of $Z^{2}\left(E \times G_{p}, U(F)\right)$ and that $\varphi(f)=f_{1} f_{2}$. Since $\varphi$ is an epimorphism we may conclude that $\bar{\varphi}$ is an epimorphism.

It remains to show that $\bar{\varphi}$ is a monomorphism. Since the order of each element of $G_{p}$ is a $p^{t h}$ power, and $E$ and $G_{p}$ commute element-wise in $E \times G_{p}$, we know by Prop. A. 1 that $f(\sigma, \tau)=f(\tau, \sigma)$ for each $\sigma$ in $E$ and $\tau$ in $G_{p}$ where 
$f$ is any element of $Z^{2}\left(E \times G_{p}, U(F)\right)$.

We next prove that for each element $f$ of $Z^{2}\left(E \times G_{p}, U(F)\right)$ there exists an element $\hat{f}$ of $Z^{2}\left(E \times G_{p}, U(F)\right)$ cohomologous to $f$ and such that $\hat{f}\left(\sigma_{1} \tau_{1}, \sigma_{2} \tau_{2}\right)=\hat{f}\left(\sigma_{1}, \sigma_{2}\right) \hat{f}\left(\tau_{1}, \tau_{2}\right)$ where $\sigma_{1}$ and $\sigma_{2}$ are in $E$ and $\tau_{1}$ and $\tau_{2}$ are in $G_{p}$. Since each element of $E \times G_{p}$ can be written uniquely in the form $\sigma \tau$ with $\sigma$ in $E$ and $\tau$ in $G_{p}$ we can define a map $\phi: E \times G_{p} \rightarrow U(F)$ by $\phi(\sigma \tau)=f(\sigma, \tau)$. Now define the 2-cocycle $\hat{f}$ by $\hat{f}(\rho, \omega)=f(\rho, \omega) \phi(\rho) \phi(\omega) / \phi(\rho \omega)$ for $\rho$ and $\omega$ in $E \times G_{p}$. Note that $\hat{f}(\sigma, \tau)=1$ whenever $\sigma$ is in $E$ and $\tau$ is in $G_{p}$, since $\phi(\sigma \tau)=f(\sigma, \tau)$ and $\phi(\sigma)=\phi(\tau)=1$. We proceed to verify that $\hat{f}$ has the desired property. Now $\hat{f}\left(\sigma_{1} \tau_{1}, \sigma_{2} \tau_{2}\right)=\hat{f}\left(\sigma_{1} \tau_{1} \sigma_{2}, \tau_{2}\right) \hat{f}\left(\sigma_{1} \tau_{1}, \sigma_{2}\right)$ since $\hat{f}\left(\sigma_{2}, \tau_{2}\right)=1$, so that it suffices to prove that $\hat{f}\left(\sigma_{1} \tau_{1} \sigma_{2}, \tau_{2}\right)=\hat{f}\left(\tau_{1}, \tau_{2}\right)$ and $\hat{f}\left(\sigma_{1} \tau_{1}, \sigma_{2}\right)=\hat{f}\left(\sigma_{1}, \sigma_{2}\right)$. The equality $\hat{f}\left(\sigma_{1} \sigma_{2} \tau_{1}, \tau_{2}\right) \hat{f}\left(\sigma_{1} \sigma_{2}, \tau_{1}\right)=\hat{f}\left(\sigma_{1} \sigma_{2}, \tau_{1} \tau_{2}\right) \hat{f}\left(\tau_{1}, \tau_{2}\right)$ implies that $\hat{f}\left(\sigma_{1} \tau_{1} \sigma_{2}, \tau_{2}\right)=\hat{f}\left(\sigma_{1} \sigma_{2} \tau_{1}, \tau_{2}\right)=\hat{f}\left(\tau_{1}, \tau_{2}\right)$ since $\hat{f}\left(\sigma_{1} \sigma_{2}, \tau_{1}\right)=\hat{f}\left(\sigma_{1} \sigma_{2}, \tau_{1} \tau_{2}\right)=1$. On the other hand, the equality $\hat{f}\left(\sigma_{1} \tau_{1}, \sigma_{2}\right) \hat{f}\left(\sigma_{1}, \tau_{1}\right)=\hat{f}\left(\sigma_{1}, \tau_{1} \sigma_{2}\right) \hat{f}\left(\tau_{1}, \sigma_{2}\right)$ implies that $\hat{f}\left(\sigma_{1} \tau_{1}, \sigma_{2}\right)=\hat{f}\left(\sigma_{1}, \tau_{1} \sigma_{2}\right)$ since $\hat{f}\left(\sigma_{1}, \tau_{1}\right)=1$ and $\hat{f}\left(\tau_{1}, \sigma_{2}\right)=\hat{f}\left(\sigma_{2}, \tau_{1}\right)=1$. But $\hat{f}\left(\sigma_{1}, \tau_{1} \tau_{2}\right)=\hat{f}\left(\sigma_{1}, \sigma_{2} \tau_{1}\right)$ and $\hat{f}\left(\sigma_{1}, \sigma_{2} \tau_{1}\right) \hat{f}\left(\sigma_{2}, \tau_{1}\right)=\hat{f}\left(\sigma_{1} \sigma_{2}, \tau_{1}\right) \hat{f}\left(\sigma_{1}, \sigma_{2}\right)$ together imply that $\hat{f}\left(\sigma_{1} \tau_{1}, \sigma_{2}\right)=\hat{f}\left(\sigma_{1}, \sigma_{2}\right)$. Therefore $\hat{f}\left(\sigma_{1} \tau_{1}, \sigma_{2} \tau_{2}\right)=\hat{f}\left(\sigma_{1}, \sigma_{2}\right) \hat{f}\left(\tau_{1}, \tau_{2}\right)$.

Now we may prove that $\bar{\varphi}$ is a monomorphism. For suppose that $f$ is a 2-cocycle for which $\bar{\varphi}([f])=[1]$. Let $\hat{f}$ be the 2-cocycle cohomologous to $f$ and satisfying $\hat{f}\left(\sigma_{1} \tau_{1}, \sigma_{2} \tau_{2}\right)=\hat{f}\left(\sigma_{1}, \sigma_{2}\right) \hat{f}\left(\tau_{1}, \tau_{2}\right)$, whose existence is established by the above. Then the fact that $\bar{\varphi}([\hat{f}])=[1]$ implies that $\left[\hat{f}_{1}\right]=[1]$ and $\left[\hat{f}_{2}\right]=[1]$. Let $\phi_{1}: E \rightarrow U(F)$ and $\phi_{2}: G_{p} \rightarrow U(F)$ be maps such that $\hat{f}_{1}\left(\sigma_{1}, \sigma_{2}\right)$ $=\phi_{1}\left(\sigma_{1}\right) \phi_{1}\left(\sigma_{2}\right) / \phi_{1}\left(\sigma_{1} \sigma_{2}\right)$ and $\hat{f}_{2}\left(\tau_{1}, \tau_{2}\right)=\phi_{2}\left(\tau_{1}\right) \phi_{2}\left(\tau_{2}\right) / \phi_{2}\left(\tau_{1} \tau_{2}\right)$ where the $\sigma_{i}$ are in $E$ and the $\tau_{i}$ are in $G_{p}$. Then the map $\phi: E \times G_{p} \rightarrow U(F)$ defined by $\phi(\sigma \tau)=\phi_{1}(\sigma) \phi_{2}(\tau)$ for $\sigma$ in $E$ and $\tau$ in $G_{p}$ satisfies $\hat{f}\left(\sigma_{1} \tau_{1}, \sigma_{2} \tau_{2}\right)=\phi\left(\sigma_{1} \tau_{1}\right) \phi\left(\sigma_{2} \tau_{2}\right) / \phi\left(\jmath_{1} \tau_{1} \sigma_{2} \tau_{2}\right)$ from which it follows that $[f]=[1]$.

The following statement follows immediately from Cor. A.2.

Corollary A.3. Let $F$ be a field of characteristic $p \neq 0$, and let $C=E_{1} \times \cdots \times E_{t}$ be a direct product of cyclic p-groups. If $C$ acts trivially on $F$, then the natural map

$$
H^{2}(C, U(F)) \rightarrow H^{2}\left(E_{1}, U(\dot{F})\right) \times \cdots \times H^{2}\left(E_{t}, U(F)\right)
$$

induced by the restriction maps is an isomorphism.

Definition. Let $C=E_{1} \times \cdots \times E_{t}$ be an Abelian $p$-group which acts trivially 
on a field $F$ of characteristic $p$. An element $f$ of $Z^{2}(C, U(F))$ of the form $f=f_{1} \cdots f_{t}$ where each element $f_{i}$ of $Z^{2}\left(E_{i}, U(F)\right)$ is normalized in the sense of cyclic groups (see p. 83 of [1]) is said to be normalized in the sense of Abelian p-groups.

According to Cor. A.3 we may always assume that an element $f$ of $Z^{2}(C, U(F))$ has been normalized in the sense of Abelian $\dot{p}$-groups.

Corollary A.4. Let $G$ be a group which acts trivially on a field $F$ of characteristic $p \neq 0$. If the subgroup $D$ of $G$ is an Abelian $p$-group, then for each element $f$ of $Z^{2}(G, U(F))$ there exists an element $f^{\prime}$ of $Z^{2}(G, U(F))$ cohomologous to $f$ and such that the restriction of $f^{\prime}$ to $D \times D$ is normalized in the sense of Abelian p-groups.

Proof. For convenience of notation let $f_{D}$ denote the restriction of $f$ to $D \times D$. By Cor. A. 3 there exists a 2-cocycle $f_{D}^{\prime}$ of $Z^{2}(D, U(F))$ cohomologous to $f_{D}$ such that $f_{D}^{\prime}$ is normalized in the sense of Abelian p-groups. Let $\phi_{D}: D \rightarrow U(F)$ be the map satisfying $f_{D}^{\prime}(\sigma, \tau)=f_{D}(\sigma, \tau) \phi_{D}(\sigma) \phi_{D}(\tau) / \phi_{D}(\sigma \tau)$ for $\sigma$ and $\tau$ in $D$. Extend $\phi_{D}$ to a map $\phi: G \rightarrow U(F)$ by defining $\phi(\sigma)=\phi_{D}(\sigma)$ if $\sigma$ is in $D$, and $\phi(\sigma)=1$ if $\sigma$ is in $G-D$. Then the element $f^{\prime}$ of $Z^{2}(G, U(F))$ defined by $f^{\prime}(\sigma, \tau)=f(\sigma, \tau) \phi(\sigma) \phi(\tau) / \phi(\sigma \tau)$ has the desired properties.

Corollary A.5. Let $F$ be a perfect field of characteristic $p \neq 0$, and $C$ an Abelian p-group which acts trivially on $F$. Then $H^{2}(C, U(F))=(1)$.

Proof. Let $C=E_{1} \times \cdots \times E_{t}$ be a decomposition of $C$ into a direct product of cyclic $p$-groups. By Cor. A.3 it suffices to show that $H^{2}\left(E_{i}, U(F)\right)=(1)$ for each $i$. So consider an element $[f]$ of $H^{2}\left(E_{i}, U(F)\right)$ and let $a$ be an element of $U(F)$ such that $[f]$ corresponds to $a \bmod [U(F)]^{e_{i}}$ under the canonical identification $H^{2}\left(E_{i}, U(F)\right)=U(F) /[U(F)]^{e_{i}}$ where $e_{i}$ denotes the order of $E_{i}$. Since $F$ is a perfect field of characteristic $p$, it follows that $a$ is an $e_{i}^{t h}$ power. Therefore $a \equiv 1 \bmod [U(F)]^{e_{i}}$ and $H^{2}\left(E_{i}, U(F)\right)=(1)$.

The following lemma shall be useful in proving a statement concerning the exactness of a sequence of cohomology groups.

LemMA A.6. Let $G$ be a p-group and $F$ a field of characteristic p upon which $G$ acts trivially. Then $Z^{1}(G, U(F))=(1)$.

Proof. Let $f: G \rightarrow U(F)$ be an element of $Z^{1}(G, U(F))$. We show first 
that $f(1)=1$. For by the associativity property of $f$ together with the fact that $G$ acts trivially on $F$ we obtain the equality $f(1)=[f(1)]^{2}$ so that $f(1)=1$. Now let $\sigma$ denote any element of $G$ and let $p^{t}$ denote the order of $\sigma$. Then $1=f(1)=f\left(\sigma^{p^{l}}\right)=[f(\sigma)]^{p^{t}}$ so that $f(\sigma)=1$ since $F$ has characteristic $p$. Therefore $f=1$, and so $Z^{1}(G, U(F))=(1)$.

Proposition A.7. Let $F$ be a field of characteristic $p \neq 0$, and $G_{p}$ a normal subgroup of a group $G$. If $G_{p}$ is a p-group which acts trivially on $F$ then the sequence

$$
(1) \rightarrow H^{2}\left(G / G_{p}, U(F)\right) \rightarrow H^{2}(G, U(F)) \rightarrow H^{2}\left(G_{p}, U(F)\right)
$$

is exact where the maps are inflation and restriction.

Proof. Since $G_{p}$ is a $p$-group and $F$ has characteristic $p$, we know by Lemma A. 6 that $H^{1}\left(G_{p}, U(F)\right)=(1)$. It now follows from Prop. 5 p. 126 of [5] that the above sequence is exact.

\section{REFERENCES}

[1] E. Artin, C. Nesbitt and R. Thrall, Rings with Minimum Condition, Michigan (1955).

[2] M. Auslander and O. Goldman, Maximal orders, Trans. Amer. Math. Soc., vol. 97 (1960), pp. 1-24.

[ 3 ] M. Auslander and D. S. Rim, Ramification index and multiplicity, Ill. J. of Math., vol. 7 (1963), pp. 566-581.

[4] M. Hall, The Theory of Groups, The Macmillan Co. (1959).

[ 5 ] J. P. Serre, Corps Locaux, Paris, Hermann (1962).

[6] E. Weiss, Algebraic Number Theory, McGraw-Hill Co. (1963).

[ 7 ] S. Williamson, Crossed products and hereditary orders, Nagoya Math. J., vol. 23 (1963), pp. $103-120$.

[ 8 ] S. Williamson, Crossed products and maximal orders, Nagoya Math. J., vol. 25 (1965), pp. 165-174.

[9] C. Curtis and I. Reiner, Representation Theory of Finite Groups andAssociative Algebras, Wiley and Sons (1962).

[10] M. Harada, Some criteria for hereditarity of crossed products, Osaka J. Math., vol. 1 (1964), pp. $69-80$.

Regis College

Weston, Massachusetts 